\title{
Research journey of respirasome
}

\author{
Meng $\mathrm{Wu}^{1}$, Jinke $\mathrm{Gu}^{1}$, Shuai Zong ${ }^{1}$, Runyu Guo ${ }^{1}$, Tianya Liu ${ }^{1}$, Maojun Yang ${ }^{1,2 \bowtie}$ \\ ${ }^{1}$ Ministry of Education Key Laboratory of Protein Science, Tsinghua-Peking Joint Center for Life Sciences, Beijing Advanced \\ Innovation Center for Structural Biology, School of Life Sciences, Tsinghua University, Beijing 100084, China \\ ${ }^{2}$ School of Pharmacy, Tongji Medical College, Huazhong University of Science and Technology, Wuhan 430030, China \\ $\bowtie$ Correspondence: maojunyang@tsinghua.edu.cn (M. Yang) \\ Received December 1, 2019 Accepted December 11, 2019
}

\begin{abstract}
Respirasome, as a vital part of the oxidative phosphorylation system, undertakes the task of transferring electrons from the electron donors to oxygen and produces a proton concentration gradient across the inner mitochondrial membrane through the coupled translocation of protons. Copious research has been carried out on this lynchpin of respiration. From the discovery of individual respiratory complexes to the report of the high-resolution structure of mammalian respiratory supercomplex $I_{1} I I I_{2} I V_{1}$, scientists have gradually uncovered the mysterious veil of the electron transport chain (ETC). With the discovery of the mammalian respiratory mega complex $I_{2} I I_{2} I V_{2}$, a new perspective emerges in the research field of the ETC. Behind these advances glitters the light of the revolution in both theory and technology. Here, we give a short review about how scientists 'see' the structure and the mechanism of respirasome from the macroscopic scale to the atomic scale during the past decades.
\end{abstract}

KEYWORDS electron transport chain, supercomplex organization, cellular respiration, structure of respirasome, cryo-EM, megacomplex

\section{INTRODUCTION}

Cellular respiration refers to a process during which the organic substrates undergoes a series of redox reactions within cells to produce inorganic or small molecular organic substances, releasing energy and generating adenosine triphosphate (ATP) molecules. Aerobic respiration might be the central function of mitochondria in mammalian cells, so no wonder it is precisely regulated from various aspects in response to varying cell conditions. Therefore, the disorder of its function will lead to changes in cell physiology and induce a variety of diseases, including many kinds of widely concerned neurodegenerative and inflammatory diseases such as Alzheimer's disease, Huntington's disease, Parkinson's disease, Friedreich's ataxia and so on (Sherer et al., 2002; Zeviani and Di Donato, 2004; Schapira, 2006; Pieczenik and Neustadt, 2007; Scharfe et al., 2009; Duchen and Szabadkai, 2010; Lim et al., 2010; Lax et al., 2011; Bates et al., 2012).

The oxidative phosphorylation (OXPHOS) in eukaryotic cell is the final step of aerobic cellular respiration, which occurs on the mitochondrial inner membrane. It is the main pathway of aerobic biosynthesis of ATP (Lenaz et al., 2016; Letts and Sazanov, 2017; Lobo-Jarne and Ugalde, 2018). OXPHOS in mammals is carried out by five classes of protein complexes anchored on the inner membrane of mitochondria. These complexes are relatively independent in both structure and function, including complex I (NADH: ubiquinone oxidoreductase, $\mathrm{Cl}$ ), complex II (succinate:ubiquinone oxidoreductase, CII), complex III (cytochrome $b c_{1}$ complex, CIII), complex IV (cytochrome c oxidase, CIV), and complex $\vee$ (ATP synthase, CV) (Wharton and Tzagoloff, 1962; Green and Tzagoloff, 1966; Hatefi, 1985; Papa et al., 2012; Zong et al., 2018a; Zong et al., 2018b; Gu et al., 2019). Complex I-IV are also called respiratory chain complexes, or electron transport chain (ETC) complexes, because they only participate in the process of electron transportation and oxygen consumption.

OXPHOS in essense is the transfer of electrons along the ETC to the oxygen and the translocation of protons from the mitochondrial matrix (MM) into the intermembrane space (IMS), which forms the electrochemical gradient of proton. The ATP molecules are synthesized by CV, as protons diffuse back into the MM. During this process, $\mathrm{Cl}, \mathrm{CIII}$ and $\mathrm{CIV}$ act as proton pumps, while CII does not.

The transfer pathway of electrons in ETC could be described as such: $\mathrm{Cl}$ oxidizes NADH to $\mathrm{NAD}^{+}$and reduces ubiquinone-10 $(\mathrm{Q})$ to ubiquinol-10 $\left(\mathrm{QH}_{2}\right)$. Cll could also 
reduce $Q$ to $\mathrm{QH}_{2}$ by oxidizing succinate to fumarate. $\mathrm{QH}_{2}$ is reoxidized to $Q$ by $\mathrm{CIII}$, and cytochrome $\mathrm{c}$ (cyt $\mathrm{c}$ ) is reduced in the meantime. CIV transfers the electrons from the reduced cytochrome $\mathrm{c}$ to $\mathrm{O}_{2}$ sequentially. The energy released in this process is used to generate ATP (Milenkovic et al., 2017; Hirst, 2018).

The organization of the respiratory chain complexes has been widely studied for decades. Many evidence show that the individual complexes could assemble into supercomplexes $\left(\mathrm{I}_{1} I \mathrm{I}_{2} I \mathrm{~V}_{1-2}, I \mathrm{I}_{2} I \mathrm{~V}_{1-2}\right)$, or even megacomplex $\left(\mathrm{I}_{2} \mid \mathrm{I}_{2} I \mathrm{~V}_{2}\right)$ (Schägger and Pfeiffer, 2000; Schagger and Pfeiffer, 2001; Bultema et al., 2009; Mileykovskaya et al., 2012; Greggio et al., 2017; Guo et al., 2017). These higher order complexes may be beneficial for maintaining the biochemical structure and increasing the physiological activity of the individual complexes, while improving the efficiency of electron transportation. A widely held opinion points out that the interaction between different complexes in the supercomplexe (SC) may be conducive to the possibility of the existence of the substrate channelling as functionally segmented CoQ pool. Supercomplexes could also reduce the production of the reactive oxygen species (ROS) by sequestrating reactive intermediates (Enriquez, 2016). These functionally active supramolecular structures are also known as respirasomes.

From biochemical experiments, to blue native polyacrylamide gel electrophoresis (BN-PAGE) analysis, from X-ray crystallography to cryo-electron microscopy (cryo-EM), advances in technology promote the development of how we understand the structure, the assembly, and the mechanism of the ETC complexes. Till today, the discussion is still far from ending. With the report of the high- resolution structures of mammalian respiratory supercomplex- $I_{1} I I_{2} I V_{1}\left(S C I_{1} I I_{2} I V_{1}\right)$ and the recognition of megacomplex- $\mathrm{I}_{2} \mathrm{II}_{2} I \mathrm{~V}_{2}\left(\mathrm{MCl}_{2} I \mathrm{I}_{2} I \mathrm{~V}_{2}\right)$ by us and several other groups, more details are presented, leading to the proposal of some new viewpoints and even more considerable controversy (Gu et al., 2016; Letts et al., 2016; Sousa et al., 2016; Wu et al., 2016; Guo et al., 2017; Guo et al., 2018). In this paper, we will give a brief review of the history of the research on respirasome, combined with the development of technology, and introduce some lately reported results and heatedly debated arguments.

\section{PRE-STRUCTURAL AGE, THEIR EXISTENCE AND THEIR FUNCTION}

The study of the OXPHOS system has gone through a long history. After the instructive investigation of Otto Warburg who discovered Atmungsferment, which founded the enzymatic basis for respiration, tantalizing advances emerged to clarify the molecular mechanisms of the respiratory enzymes. The term oxidative phosphorylation was first proposed by Volodymyr Belitser in 1939, who measured the P/O ratios (a division of the rate of ATP production and oxygen consumption) of OXPHOS in minced and homogenized heart muscle and pigeon breast muscle, indicating the possibility that some intermediate redox reactions are coupled with phosphorylation. During 1900s to 1930s, active work on bioenergetics were done by spectral and chromatographic analysis and biochemical experiments (Kalckar, 1974, 1991). In 1955, Britton Chanc and G.R.Williams first raised the idea that the redox enzymes and prosthetic groups that are supposed to be responsible to electron transportation could assemble into larger complexes, which were then reconstituted as electron transfer system (Chance and Williams, 1955). Four functional complexes were purified and reconstructed by Hatefi et al., till 1962, termed ClCIV (Hatefi et al., 1962). Together with the isolation of electron donors $\mathrm{NADH}$ and FAD and the identification of the roles of $Q$ and cyt c, the ETC system gradually matured (Hill and Keilin, 1930; Crane et al., 1957; Enríquez, 2016). Other redox reactions providing electrons to reduce coenzyme $Q$ such as dihydroorotate dehydrogenase (Evans and Guy, 2004), glycerol 3 phosphate dehydrogenase (Harding et al., 1975) and electron transport flavoprotein dehydrogenases (Bentinger et al., 2010; Alcázar-Fabra et al., 2016) are also found.

A question ensues, how does ETC drive ATP synthesis? The most commonly accepted theory was the chemiosmotic hypothesis raised by Peter D. Mitchell in 1961 (Mitchell, 1961). The work of Hatefi et al. provided support to the chemiosmotic hypothesis (Hatefi et al., 1962) because their purified ETC complexes showed enzymatic activities. Chemiosmotic hypothesis advocates for a reversible proton translocation ATPase system and oxido-reduction chain that could make use of the diffusion-driven force known as proton-motive force (PMF) generated by the electrochemical potential between both sides of the mitochondrial inner membrane to produce ATP. The interaction between ETC complexes and ATP synthase is unnecessary, while the battery like mitochondrial inner membrane stores the energy needed (Mitchell, 2011; Enríquez, 2016; Hirst, 2018). This is inconsistent with the previous idea that the respiratory components were rigidly coupled as a functional unit, which requires complicated regulation of stoichiometries at each step (Enríquez, 2016).

The process of how ETC complexes implement their functions is closely linked with another question: how do ETC complexes organize on the mitochondrial inner membrane? Three models have been proposed successively: the solid model, fluid model (also known as random diffusion model) and plasticity model. The solid model proposed by Keilin, Chance et al. regarded ETC catalytic complexes as solidly associated single units that can catalyze whole reaction pathways, with their electron carriers also enclosed in the pathways allowing for a better electron transporting efficiency (Keilin and Hartree, 1947; Chance and Williams, 1955; Chance et al., 1963; Blair, 1967; Lenaz and Genova, 2007; Acin-Perez et al., 2008). Whereas in the fluid model, individual ETC complexes and redox components move in diffusional motion constantly and independently in the membrane, and the electrons are transferred between the 
complexes through the free diffusion of $Q$ and cyt c (Hackenbrock, 1977). The fluid model gained general recognition for decades and was consistent with the chemiosmotic hypothesis (Hatefi et al., 1962; Kroger and Klingenberg, 1973; Hochli and Hackenbrock, 1976; Hochli et al., 1985; Gupte and Hackenbrock, 1988). However, as time went on, neither of these two models could withstand all of the surfacing experimental evidence.

\section{ERA OF X-RAY CRYSTALLOGRAPHY, AN UNREMITTING PURSUIT}

Structural biology has been an important subject that enables people to understand the structure of biological molecules in great detail. Multiple methodologies derived from different mathematical and physical principles have been exploited to determine the spatial relationship of the atoms within biological molecules. The most widely implemented methods include nuclear magnetic resonance (NMR) spectroscopy, X-ray crystallography, neutron diffraction, cryo-EM, and spectroscopic techniques. X-ray crystallography has been the most powerful tool in resolving structures of macromolecules at atomic resolution since its birth over a century ago (Shi, 2014; Powell, 2017; Wang and Wang, 2017; Standfuss, 2019). More than 138,000 molecular structures, reported with this technique, have been deposited in the Protein Data Bank (PDB) since its establishment in 1971, endowing X-ray crystallography with the dominant role in structural biology. From the late 1960s, research on ETC has begun to incorporate structural biological techniques.

The first successful case of structural analysis of ETC complexes was the $2.8 \AA$ crystal structures of CIV extracted from bacterial and bovine heart published in 1995 and 1996 (Iwata et al., 1995; Tsukihara et al., 1995, 1996). CIV in crystal structures existed as dimers with each monomer consisting of 13 different subunits. In contrast, we proved CIV is a 14-subunit monomer in native state recently (Zong et al., 2018b). In 1998, the precise locations of its cofactors, heme $\mathrm{a}$, heme $\mathrm{a}_{3}, \mathrm{Cu}_{\mathrm{A}}$ and $\mathrm{Cu}_{\mathrm{B}}$ were identified (Brzezinski and Adelroth, 1998). In the year 2012, Eduardo Balsa and partners pointed out that NDUFA4 which was considered to be a subunit of $\mathrm{Cl}$ was actually the subunit of CIV (Balsa et al., 2012).

It is now recognized that mammalian CIV is composed of three core subunits encoded by mitochondrial genes (COXI, COXII and COXIII) and eleven subunits encoded by nuclear genes. Cofactors heme a (containing $\mathrm{Fe}_{\mathrm{a}}$ ), heme $\mathrm{a}_{3}$ (containing $\mathrm{Fe}_{\mathrm{a} 3}$ ) and $\mathrm{Cu}_{\mathrm{B}}$ are located in subunit $\mathrm{COXI}$, with heme $\mathrm{a}_{3}\left(\mathrm{Fe}_{\mathrm{a} 3}\right)$ and $\mathrm{Cu}_{\mathrm{B}}$ forming a binuclear center. $\mathrm{Cu}_{\mathrm{A}}$ is in subunit COXII. These four redox-active metal centers constitute an electron transport pathway. In each catalytic cycle, $\mathrm{Cu}_{\mathrm{A}}$ accepts electrons donated from four reduced cyt $\mathrm{c}$ consecutively and transfer them to the active site through $\mathrm{Fe}_{\mathrm{a}}$. Two protons are taken from MM with one electron transported to the $\mathrm{Fe}_{\mathrm{a} 3} / \mathrm{Cu}_{\mathrm{B}}$ binuclear center. In total, eight protons are uptaken from $\mathrm{MM}$, with four of them used to produce $\mathrm{H}_{2} \mathrm{O}$ and four pumped into IMS to form the proton gradient (Konstantinov et al., 1997; Brzezinski and Adelroth, 1998; Sousa et al., 2018). Three channels for proton transportation named D-, K-, and $\mathrm{H}$-channel have been described (Wikstrom et al., 2015). The K- channel is responsible for the transportation of two protons consumed in the reduction of $\mathrm{O}_{2}$ to $\mathrm{H}_{2} \mathrm{O}$ from MM to the binuclear center for water formation. The D- channel conducts the other two protons consumed for the reduction of $\mathrm{O}_{2}$, and provide the pathway for the four protons pumped into the IMS (Konstantinov et al., 1997; Brzezinski and Adelroth, 1998). The H- channel consist of water cavities and polar residues, and is supposed to be functionally associated with a hydrogen- bond network linked to oxido-reduction of heme a (Yoshikawa et al., 1998; Yoshikawa and Shimada, 2015; Papa et al., 2018).

CIII was the second member to get its crystal structure in ETC complexes. The first complete structure of CIII was obtained in 1998 (Iwata et al., 1998). Iwata et al. purified CIV from bovine heart and reported its atomic structure as a symmetrical homodimer consisting of 22 subunits in total. However, we found CIII is actually an asymmetric 21-subunit dimer in 2018 (Zong et al., 2018a). The mammalian CIII monomer is composed of three highly conserved core subunits and eight supernumerary subunits. The core subunits include a mitochondrial encoded cytochrome b (cyt b) with heme $b_{L}$, heme $b_{H}$ and two distinct quinone-binding sites, $a$ nuclear-encoded cytochrome $c_{1}$ (cyt $c_{1}$ ) with heme $c_{1}$, and a Rieske iron-sulfur protein (ISP) with a [2Fe-2S] cluster (Yang and Trumpower, 1986). Two quinone-binding sites named $Q_{o}$ and $Q_{i}$ in cyt b locate on opposite sides of mitochondrial inner membrane (Xia et al., 1997; Pietras et al., 2016). A feature of CIII dimer is that each ISP subunit of a monomer spans two monomers with the transmembrane domain associating with one monomer while the soluble domain remains in the other monomer. It is still controversial whether the two monomers of $\mathrm{Cll}_{2}$ function cooperatively or independently.

Crystal structures of Cll were first studied in prokaryote. Iverson and partners reported the $3.3 \AA$ structure of Escherichia coli fumarate reductase (QFR) (Iverson et al., 1999). In 2003, Yankovskaya et al. reported the structure of CII (SQR) (Yankovskaya et al., 2003). The first mammalian Cll crystal structure at a resolution of $2.4 \AA$ was determined in 2005 with porcine heart (Sun et al., 2005). Cll is composed of an FAD binding protein (flavoprotein,Fp), an iron-sulfur protein (Ip) and two membrane-anchor proteins (CybL and CybS). Fp and Ip form the hydrophilic head, while CybL and CybS form the hydrophobic arm. Three kinds of prosthetic groups, FAD, heme and iron-sulfur clusters, were recognized in Cll, coupled with two $Q$-binding sites $\left(Q_{P}\right.$ and $\left.Q_{D}\right)$. Herein, Fp contained the FAD cofactor, Ip contained three iron-sulfur clusters ([2Fe-2S], [4Fe-4S] and [3Fe-4S]), yet CybL and CybS each had a heme b (Cecchini, 2003; Bezawork-Geleta et al., 2017). The Q-binding sites were investigated by 
means of mutagenesis and kinetic analysis with inhibitors. $Q_{P}$ site is proximal to the matrix side of inner mitochondrial membrane (IMM), and $Q_{D}$ site is distal from the matrix. During the succinate oxidation reaction, two electrons are transferred from the falvin to reduce $Q$ bound at $Q_{P}$ via the iron-sulfur clusters [2Fe-2S], [4Fe-4S] and [3Fe-4S]. There is little categorical data explicating the role of the heme and $Q_{D}$ site (Bezawork-Geleta et al., 2017; Sousa et al., 2018).

$\mathrm{Cl}$ is the largest and most complicated protein complex in ETC and is vital to cellular metabolism. In many eubacteria, this type of enzyme is termed as NADH dehydrogenase-1 or $\mathrm{NDH}-1$. The sodium-pumping $\mathrm{NADH}$-quinone reductase $\left(\mathrm{Na}^{+}-\mathrm{NQR}\right)$ and the type II NAD(P)H dehydrogenase (NDHII) are also members of this protein family (Melo et al., 2004; Barquera, 2014). In plants, many fungi and many bacteria, four so-called alternative NADH dehydrogenases are found, which do not couple the redox reaction to proton or sodium translocation (Kerscher, 2000; Brandt, 2006; Kerscher et al., 2008; Sousa et al., 2018). After its purification from bovine heart in 1962, the molecular structure of $\mathrm{Cl}$ remained elusive for a long time. The architectures of $\mathrm{Cl}$ were determined with electron microscopy at the early stage (Leonard et al., 1987; Hofhaus et al., 1991; Grigorieff, 1998; Peng et al., 2003; Radermacher et al., 2006). It was not until 2010 that scientists resolved the first crystal structure of the entire $\mathrm{Cl}$ from Y. lipolytica at a resolution of $6.3 \AA$ (Hunte et al., 2010). However, a complete atomic resolution structure obtained with X-ray crystallography is still lacking.

Structural analysis of $\mathrm{Cl}$ in this era indicates that with a molecular mass of about $970 \mathrm{kDa}$, integral mammalian $\mathrm{Cl}$ is composed of 45 subunits assembled into an L-shaped architecture. The minimal functional unit of mammalian $\mathrm{Cl}$ comprises 14 subunits known as core subunits. Subunits ND1-ND6 and ND4L are encoded by mitochondrial genome and form the hydrophobic domain contained in the mitochondrial inner membrane. The other seven core subunits form the hydrophilic arm comprising a flavin mononucleotide (FMN) and eight iron-sulfur clusters as redox active prosthetic groups and extends into the MM. Up to 31 supernumerary subunits (include two NDUFAB1 subunits) are identified in the intact mammalian $\mathrm{Cl}$. These subunits play an important role in the assembly, stabilization and regulation of $\mathrm{Cl}$ and fulfill the independent function of mitochondrial metabolism. In $\mathrm{Cl}$, two electrons are transferred from $\mathrm{NADH}$ to $\mathrm{FMN}$ and then to quinone via seven iron-sulfur clusters (N3, N1b, N4, N5, N6a, N6b, and N2). Cluster N2 is the direct reductant for quinone. Cluster $\mathrm{N} 1 \mathrm{a}$ may play a role in preventing the excessive production of ROS (Sazanov et al., 2013; Friedrich, 2014; Sazanov, 2015). Four protons are translocated into the IMS during this process. Many hypothetical mechanisms have been proposed to clarify the coupling between electron and proton transfers. Evidence suggest that long-range conformational change may be related to this process. One or two-stroke mechanisms have been discussed. One-stroke mechanism offers a model in which four protons are translocated all at once, driven by the redox of one quinone molecule. The two-stroke model, on the other hand, proposes that two sequential one-electron $Q$ reduction steps induce two conformational changes, each translocating two protons (Brandt, 2011; Efremov and Sazanov, 2012; Hirst, 2013).

Aside from $\mathrm{Cl}$, in various fungi, plants, and primitive cells, an alternative enzyme was found able to reduce $Q$, but lacking the proton pumping capacity. This alternative enzyme, NDH2 (NADH dehydrogenase type-II), was first identified in domestic yeast as a $50 \mathrm{kDa}$ single-subunit membrane protein (Ohnishi et al., 1966), and was shown to be essential for the long-term survival of some pathogenic microorganisms (Biagini et al., 2012; Verner et al., 2013; Yano et al., 2014), like P. falciparum, Toxoplasma gondi, and Mycobacterium tuberculosis. In 2012, our group solved the X-ray structures of Ndi1 (NDH2 from yeast) in apo, $\mathrm{NADH}-$, $\mathrm{Q}-$, and $\mathrm{NADH}-\mathrm{Q}-$ bound states and demonstrated that electron transfer in $\mathrm{NDH} 2$ requires two $\mathrm{Q}$ molecules and CTD of NDH2 mediates the homodimerization and membrane attachment (Feng et al., 2012). Later on, our group reported the X-ray structures of PfNDH2 (NDH2 from $P$. falciparum) in its apo, NADH-, and RYL-552 (a new inhibitor)bound states, unveiling the inhibiting mechanism of $P$ NDH2 and providing accurate information for designing new antimalarial drugs (Yang et al., 2017).

The X-ray crystallography studies of ETC complexes provide important structural information for the study of respiration. However, the biostructural research of ETC complexes is far from perfect. In the age of cryo-EM today, new exciting discoveries are emerging, helping build the edifice of respiratory system.

\section{IDENTIFICATION OF RESPIRASOME AND THE PLASTICITY MODEL}

The crystal structures of individual ETC complexes are gradually reported with the efforts of researchers. However, the investigation on the functional mechanism of ETC is still filled with dark clouds. The existence of higher-order organization of ETC complexes has been considered for a long time. The invention of BN-PAGE technology created a new climate to the debates. BN-PAGE can be used to isolate protein complexes and determine their native protein masses and oligomeric states. The first BN-PAGE analysis was carried out by Schägger and Pfeiffer in 2000 with digitoninsolubilized mitochondrial extracts from yeast and bovine, revealing the co-migration of respiratory complexes in sucrose gradient centrifugation and in gel (Schägger and Pfeiffer, 2000). This finding led to the revival of the solid model. The concept of respirasome was then proposed. Respirasome was originally considered to have a fixed composition as two copies of $\mathrm{SCl}_{1} \mathrm{II}_{2} \mathrm{IV}_{4}$ and one copy of $\mathrm{SCIII}_{2} \mathrm{IV}_{4}$ to consist with the overall 1:3:6 stoichiometric ratio of complexes I:III:IV (Schägger and Pfeiffer, 2000; Wittig et al., 2006a). However, these kinds of entities were not 
detected. Contrarily, superassemblies as $\mathrm{I}_{1} \mathrm{II}_{2}, \mathrm{III}_{2} \mathrm{IV} \mathrm{V}_{1}$ and free complexes were observed. Henceforth, although accompanied with significant skepticism, SCs from different sources such as yeast and other fungi, plants, vertebrates, and invertebrates were found by BN-PAGE with a variety of detergents (Eubel et al., 2004; Krause et al., 2004a; Krause et al., 2004b; Stroh et al., 2004; Krause, 2006; Reifschneider et al., 2006; Marques et al., 2007; Bultema et al., 2009). The co-migration on sucrose gradients also indicates the existence of SCs. Another convincing proof is the detection of SCs in the tomograms of cristae isolated from Podospora anserine (Davies et al., 2011). Recent subtomogram averaging results give a further confirmation of the ubiquitous existence of respirasomes (Davies et al., 2018). In vitro experiments suggest that the isolated respirasomes still possess the ability to transfer electrons from NADH to oxygen (Acin-Perez et al., 2008; Milenkovic et al., 2017). The stability of $\mathrm{Cl}$ is dependent on $\mathrm{CIII}_{2}$ and CIV (Schagger et al., 2004; Stroh et al., 2004).

The majority of $\mathrm{Cl}$ is found to assemble into SCs with CIII and CIV, while CII tends to exist in a non-associated form in plant and mammalian mitochondria (Schagger and Pfeiffer, 2001). The most common forms of SCs include $\mathrm{SCl}_{1} \mathrm{III}_{\mathrm{n}}$, $\mathrm{SCl}_{1} \mathrm{II}_{2 \mathrm{~m}} \mathrm{IV}$ and $\mathrm{SCII}_{2 \mathrm{~m}} \mathrm{IV}_{\mathrm{n}}$ (Maranzana et al., 2013). The most interesting one among which is $S_{C l} I_{1} I_{2} I V_{1}$ (Fig. 1A), because this supercomplex contains all components required to accomplish the electron transportation, from $\mathrm{NADH}$ to oxygen together with cyt $\mathrm{c}$ and quinone, and is conserved in mammalian mitochondria (Schägger and Pfeiffer, 2000; Lenaz and Genova, 2012). Only a few cases hypothesize the association of Cll.

The fact that both free and diversely superassembled respiratory complexes are able to partake in cellular respiration has spawned a proposal called plasticity model. This view suggests a dynamic situation where the respiratory complexes can exist and function in both free and diversely superassembled modes. The stoichiometries and stabilities of free and superassembled complexes may vary with cell types and physiological stimuli (Acin-Perez et al., 2008; Lenaz and Genova, 2012; Enríquez, 2016). This dynamic equilibrium state is supported by some indirect evidence (Gomez et al., 2009; Frenzel et al., 2010; Hofmann et al., 2012). Starvation may reduce the amount of $S \mathrm{SI}_{1} \mathrm{II}_{2} \mathrm{IV}_{1}$ in mice liver mitochondria (Lapuente-Brun et al., 2013). Hypoxia induces the dissociation of the $\mathrm{SCl}_{1} \mathrm{III}_{2} \mathrm{IV}_{1}$ into free $\mathrm{Cl}$ and the $\mathrm{SCIII} / \mathrm{IV}$ in potato, while the expression of alternative oxidase (AOX, a $\mathrm{Q}$ oxidase found in plants and fungi, but not found in most animals) can stable free $\mathrm{Cl}$ without the presence of CIII and IV (Ramirez-Aguilar et al., 2011). The validity of the reversible dynamic association/dissociation between ETC complexes and supercomplexes remains unproven.

\section{CRYO-EM, OLD TREES AND NEW BUDS}

The ability to purify SCs from mitochondria allows for their visualization and structural determination at low resolutions. As a promising technology gaining great attention in recent years, cryo-EM has come to exhibit its latent capacity in the field of structural biology. The advantages of cryo-EM are as follows: less sample requirement, no need for crystallization, less limitation on sample purity, and suitable for the structural analysis of biological molecules or complexes with larger molecular weight. However, 3D reconstruction using cryoEM is by no means a newborn methodology.

In 1926, German physicist Hans Busch put forward the idea of using magnetic field generated in the short coil of a cathode ray tube to condense electron beam and use it for imaging. Based on this theory, Ruska, together with partners, developed the first EM in 1930s and was awarded the Nobel Prize in Physics in 1986 (Robinson, 1986).

The history of analyzing the structure of biological macromolecules by transmission electron microscopy can be traced back to 1960s. DeRosier and Klug published the first 3D EM structure of the tail of bacteriophage T4 and formulated the general principles of $3 D$ reconstruction tack using helical Fourier inversion method simultaneously (De Rosier and Klug, 1968). To avoid the radiation damage caused by the beam induced breakage of chemical bonds, Dubochet and colleagues developed the method of freezing the samples into a thin layer of amorphous ice (also referred to as vitreous ice) (Dubochet et al., 1981; Adrian et al., 1984; Lepault et al., 1987; Dubochet et al., 1988) based on the early study of Henderson and Unwin as well as that of Taylor and Glaeser (Taylor and Glaeser, 1974; Henderson and Unwin, 1975; Hayward and Glaeser, 1979; Bai et al., 2015). The samples are stored and imaged at the temperature of liquid nitrogen; hence, the naming "cryo-EM". The vitrified samples anchor the molecules in a close-to-native environment, preserving the structural details of particles without introducing artifacts. Meanwhile, flash-frozen amorphous ice helps prevent the dehydration of biological samples in the vacuum during imaging.

Three main branches have been derived from the cryoEM technology: 2/3D electron crystallography, single particle $3 \mathrm{D}$ reconstruction, and cryo-electron tomography (cryo-ET). Electron crystallography is the first branch to reach nearatomic and atomic resolutions. However, because of the relatively harsher conditions for sample preparation and screening, the application of this method has been restricted to some extent. The more traditional way of cryo-EM structure determination is using $2 \mathrm{D}$ projections of molecules in various directions to achieve $3 \mathrm{D}$ reconstruction, based on the rationales DeRosier and Klug built with negative stained samples (De Rosier and Klug, 1968) (Fig. 2). 
A

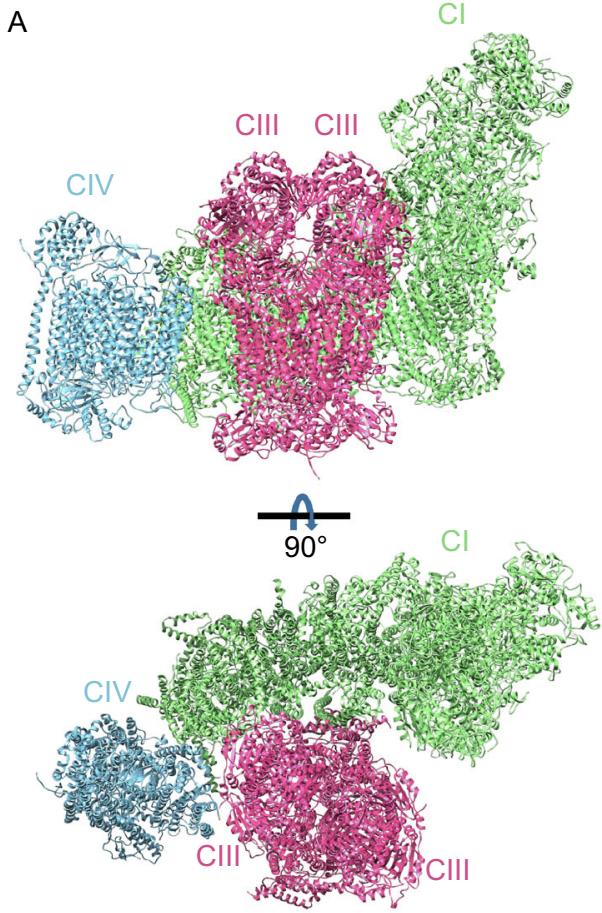

C

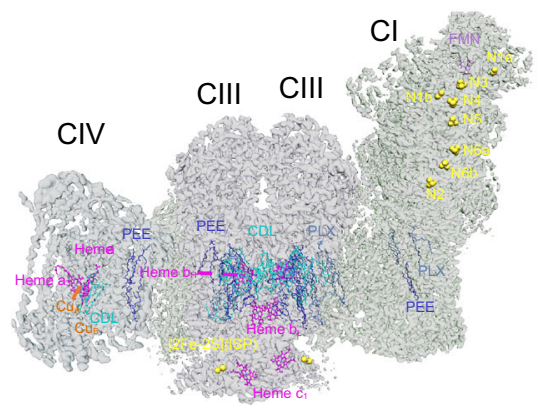

B

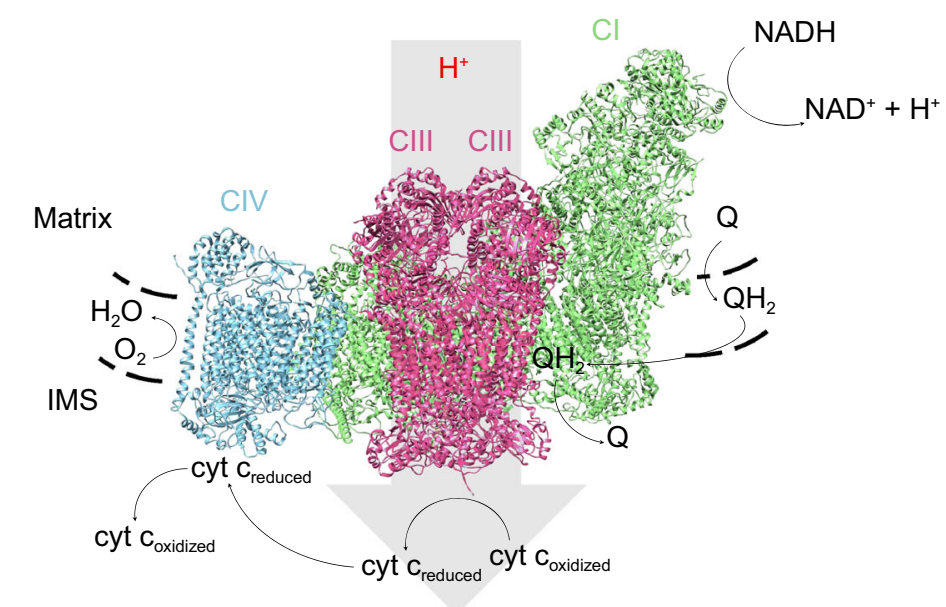

D

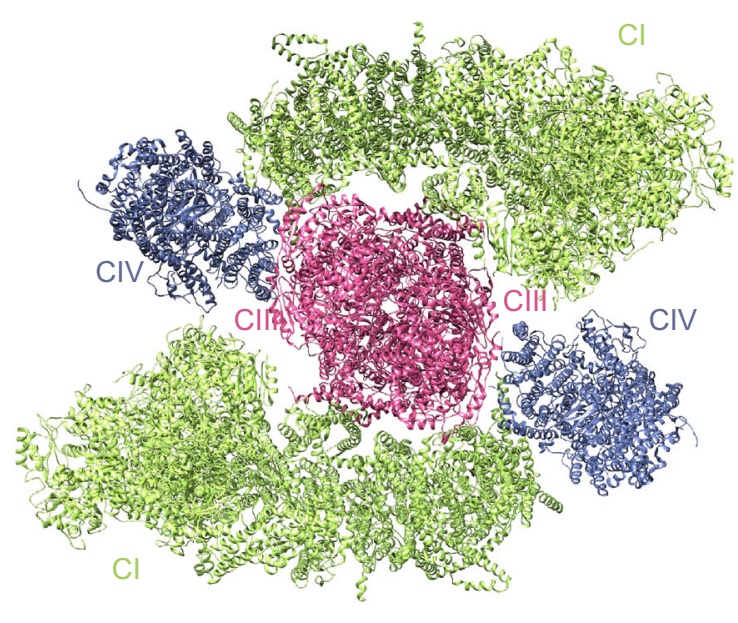

Figure 1. Structures of Mammalian $\mathrm{SCl}_{1} \mathrm{III}_{2} \mathrm{IV} \mathrm{V}_{1}$ and $\mathrm{MC} \mathrm{I}_{2} \mathrm{III}_{2} \mathrm{IV} \mathbf{V}_{2}$. (A) Cartoon representation of the cryo-EM structure of human $\mathrm{SCl}_{1} I_{2} \mathrm{IV}_{1}$ (PDB ID: $5 \mathrm{XTH}$ ) shown in two orientations. The side view along the inner membrane, and the top view perpendicular to the inner membrane. Individual $\mathrm{Cl}, \mathrm{CIII}$, and CIV monomers are labeled with texts in the same colors with the represented structures, respectively. (B) Substrate translocation in $\mathrm{SCl}_{1} \mathrm{II}_{2} \mathrm{IV}_{1}$. The black dotted lines represent mitochondrial inner membrane. The grey arrow indicates the translocation of protons. The black arrows indicate changes of substrates before and after the redox reactions. (C) Cofactors in $\mathrm{SCl}_{1} \mathrm{Il}_{2} I \mathrm{~V}_{1}$. Different cofactors are shown in different colors. Hemes are shown as stick and ball model, FeS clusters and ions are shown as spheres, and phospholipid molecules and FMN are shown as lines. The colors of the label texts are the same with colors of the represented structures. (D) Cartoon representation of the structure of human $\mathrm{MCl}_{2} \mathrm{III}_{2} \mathrm{IV} \mathrm{V}_{2}$ (PDB ID: 5XTI).

The basis of cryo-EM 3D reconstruction is the theorem named projection-slice theorem, or central/Fourier slice theorem. The theorem states that the Fourier transform of a $2 \mathrm{D}$ projection of a $3 \mathrm{D}$ object in real space is equivalent to a central 2D slice of the 3D Fourier transform of that object. The real-space projection direction is orthogonal to the slice. TEM images represent the $2 \mathrm{D}$ projections of molecules in the sample. Multiple copies of same molecules in the vitrified sample are preserved in various directions. Considering these facts synthetically, if the directions of the $2 \mathrm{D}$ projections are known, 2D Fourier slices of these projections can be positioned back into the $3 \mathrm{D}$ Fourier space; thus, the reconstruction of the origin molecular structure can be done by computing the inverse Fourier transform. The resolution 
Electron diffraction patterns

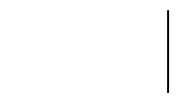

Electron crystallography

B

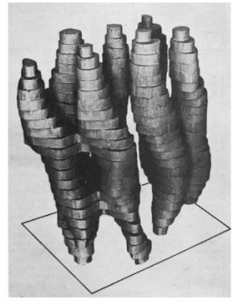

D

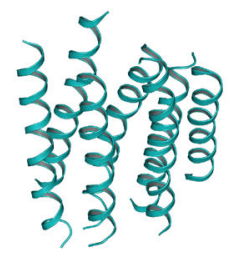

MicroED

E

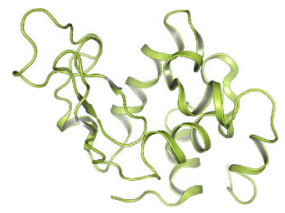

F

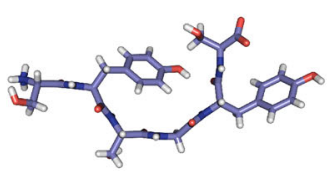

A

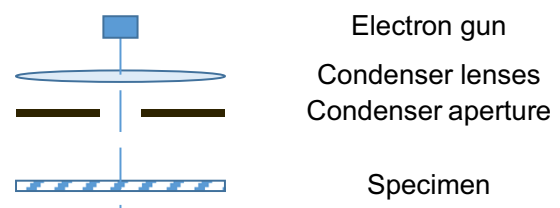

Objective lens
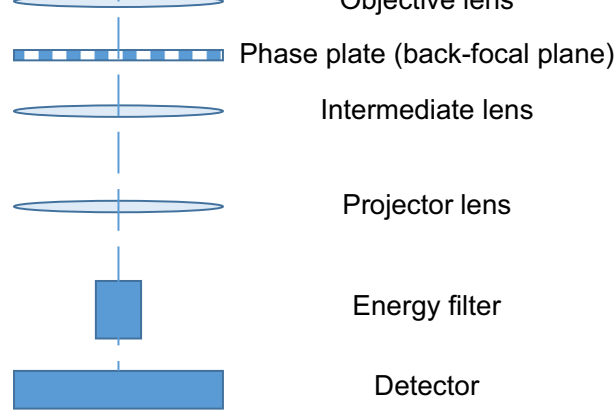

Projector lens

Energy filter

Detector

Projections of entities in multiple directions

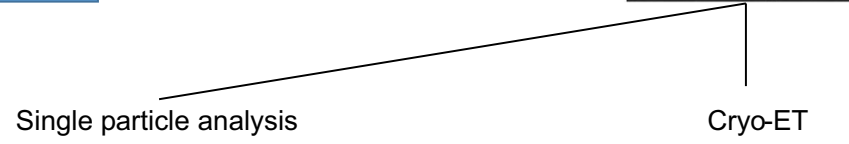

G

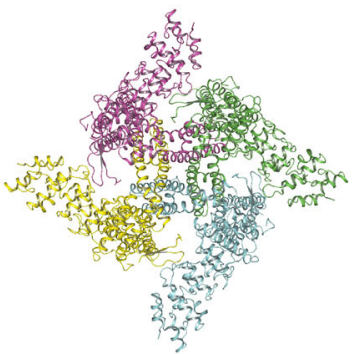

$J$

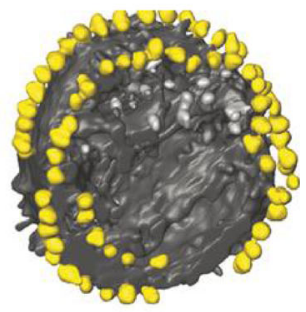

$\mathrm{H}$

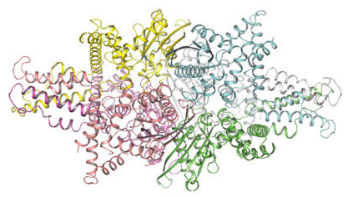

Subtomogram averaging

K

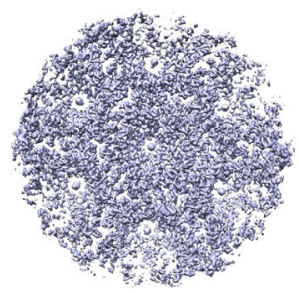

Figure 2. Evolution of EM in Structural Biology. (A) Schematic diagram of a transmission electron microscope (TEM) equipped with a phase plate, energy filter and direct electron detector. (B) The first map of bacteriorhodopsin determined by electron crystallography at a resolution of $7 \AA$ (Henderson and Unwin, 1975). The image of the map is derived from the original paper. (C) The first map of the tail of Bacteriophage T4 obtained on the basis of Fourier inversion method. The image of the map is derived from the original paper. (D) First near-atomic-resolution biological macromolecular structure of bacteriorhodopsin obtained by electron crystallography (PDB ID: 1brd) (Henderson et al., 1990). (E) $2.9 \AA$ structure of lysozyme (PDB ID: 3J4G), laying the first stone for the MicroED technique. (F) $0.7 \AA$ structure of RNA-binding protein FUS (37-42) SYSGYS segment determined by MicroED (PDB ID: 5XSG) (Luo et al., 2018). (G) $3.3 \AA$ structure of TRPV1 ion channel determined by single particle analysis method, representative near-atomic-resolution structure obtained with DDD and motion correction method (PDB ID: 3J5P) (Liao et al., 2013). (H) 1.8 $\AA$ structure of glutamate dehydrogenase (PDB ID: 5K12) (Merk et al., 2016). Published structure of highest resolution obtained with single particle analysis method. (I) $3.2 \AA$ structure of $52 \mathrm{kDa}$ biotin-bound streptavidin (PDB ID: 6J6J) (Fan et al., 2019). Smallest integral biological macromolecular structure determined at near-atomic resolution. $(\mathrm{J})$ Tomographic volumes of respiratory $\mathrm{CV}$ dimer ribbons (Strauss et al., 2008). The image of the map is derived from the original paper. (K) $3.4 \AA$ density map of HIV- 1 dMACANC VLPS (EMDB ID: emd_3782) (Turonova et al., 2017), indicating a successful trial of subtomogram averaging and 3D-CTF algorithm. 
of reconstructed results can be improved through performing multiple iterations. With sufficient number of images containing high resolution information that are accurately classified and aligned, 3D structure at atomic resolution can be obtained by single particle reconstruction.

The problem of low signal-to-noise ratio (SNR) caused by low electron dose imaging has accompanied cryo-EM technology since its birth. The loss of particle spatial orientation and position information including three Euler angles, two inplane positional parameters as well as the defocus value also hinders the reconstruction. Even though theoretical assessment suggests that single particle analysis may reach atomic resolution for molecules with a molecular weight of around $100 \mathrm{kDa}$, the reality has been far from satisfactory for a long period (Bai et al., 2015).

Tremendous efforts have been made in aspects of both hardware and algorithm. Commonly used optimization methods for commercialized EM include applying constantpower electromagnetic lenses, using field emission gun (FEG) as electron source for a better parallel illumination, adopting high vacuum, and employing better computer control for microscope tuning and data acquisition (Cheng, 2015). The most revolutionary progress for instrumentation in recent years is the advent of direct electron detection devices (DDDs). DDDs produce significantly better images than traditional film or charge-coupled devices (CCDs). DDD cameras no longer converts electronic signals into optical signals but detects electrons directly. Individual electrons are identified (Cheng, 2015; Kuijper et al., 2015; Nogales and Scheres, 2015). With the high-enough SNR and location of each electron event being determined precisely enough, DDD gives much higher detective quantum efficiency (DQE) (McMullan et al., 2014). If the arrival point of individual electrons can be determined to subpixel accuracy, DDDs might be used in super-resolution mode beyond Nyquist cutoff frequency (Kuijper et al., 2015; McMullan et al., 2016).

Another important feature of DDD cameras is their fast frame readout rate. This allows the total electron dose used to image biological samples to be split into multiple frames, making the final output into subframe-motion-correctable movies (Bai et al., 2015; Cheng, 2015). Drift correction of these frames before averaging can eliminate the beam-induced image blurring.

Many advances in image processing methods have also been made. After the concept of single particle analysis was established in 1970s, multivariate statistical analysis was introduced into the particle classification process in 1981 (van Heel and Frank, 1981). In the same year, modular software pioneered by Joachim Frank and Marin van Heel for electron image processing emerged (Frank et al., 1981; van Heel and Keegstra, 1981). Then in 1987, ab initio methods for projection angle determination termed random conical tilt and angular reconstitution (common lines) were proposed (Radermacher et al., 1987). Projection matching and angular refinement method were set up in 1994 (Penczek et al., 1994), followed by the adhibition of maximum-likelihood approach in 1998 (Sigworth, 1998). Based on these sound foundations, more algorithmic optimization methods are proposed, such as the introduction of the empirical Bayesian approach and the gold-standard approach (Scheres, 2012a, b; Scheres and Chen, 2012), stochastic gradient descent (SGD) and branch-and-bound maximum likelihood optimization algorithms (Punjani et al., 2017), the sequential Monte Carlo method based particlefilter algorithm (Hu et al., 2018), and different approaches used for automated particle picking (Nicholson and Glaeser, 2001; Adiga et al., 2004; Ogura and Sato, 2004; Wong et al., 2004; Langlois et al., 2014; Wang et al., 2016; Al-Azzawi et al., 2019b, a; Wagner et al., 2019). These explorations eventually led to the birth of the widely used semi-automated $3 \mathrm{D}$ reconstruction software.

Another thing worth noting is the motion correction of the raw data collected from cryo-EM equipped with DDD cameras. The inevitable movement of molecules during the data acquisition reduces the overall quality of the photographs and the final resolution of the reconstruction obtained from the data. To better understand and deal with beam-induced motion is one of the most challenging physical problems for cryo-EM. Beam-induced sample motion can be divided into two components, uniform whole-frame motion and idiosyncratic local motion (Brilot et al., 2012; Li et al., 2013). In 2013, an algorithm for correcting relative motion between subframes was published, in which measurements of image shifts between all frames are performed to calculate leastsquares estimates of relative shifts between adjacent frames. It provides an effective correction of whole-frame motions with sufficient accuracy for near-atomic-resolution 3D reconstructions ( $\mathrm{Li}$ et al., 2013; Zheng et al., 2017). Individual particle tracking or local motion correction methods are proposed to simulate and estimate particle trajectories and cumulative beam-induced damage, improving the resolution of 3D reconstruction (Bai et al., 2013; Scheres, 2014; Rubinstein and Brubaker, 2015; Scheres, 2016; Zheng et al., 2017; Zivanov et al., 2019).

The craft of sample preparation is another fast-developing area. The invention of semi-automated sample preparation robots is a great convenience; however, its stability and repeatability still need to be improved. Other promising technologies include the preparation of graphene supporting film (Russo and Passmore, 2016), whisker-assisted blotting (Razinkov et al., 2016; Frank, 2017a), and spraying-plunging method (Feng et al., 2017).

\section{CRYO-EM STRUCTURES OF THE MAMMALIAN RESPIRASOMES}

The 3D structure of respirasome has been studied with EM at low resolutions from 33 to $18 \AA$ (Schafer et al., 2006; Schafer et al., 2007; Althoff et al., 2011; Dudkina et al., 2011). The emergence of new technologies has triggered an upsurge in the use of cryo-EM in structural biology. This 
wave swept through broad and diverse areas, including the structural research of the ETC. In 2014, first modern cryoEM structure of bovine complex I was reported (Vinothkumar et al., 2014). 2016 is a momentous year for the study of respirasomes, with near-atomic-resolution structures of integral mammalian $\mathrm{Cl}$ and $\mathrm{SCl}_{1} \mathrm{II}_{2} \mathrm{IV}_{1}$ being reported in succession.

The publication of the $4.2 \AA$ structure of the first integral bovine-heart $\mathrm{Cl}$ opened the prelude to these exciting results (Zhu et al., 2016). In the immediate aftermath, we and Sazanov's group reported the architectures of porcine and ovine respirasome $S_{C l} I_{1} I_{2} I V_{1}$ at $5.4 \AA$ and $5.8 \AA$, respectively (Gu et al., 2016; Letts et al., 2016). The intact mammalian $\mathrm{Cl}$ structure at $3.6 \AA$ derived from $\mathrm{SCl}_{1} \mathrm{II}_{2} \mathrm{IV} \mathrm{V}_{1}$, which is the first atomic resolution structure of $\mathrm{Cl}$, was also obtained (Wu et al., 2016) shortly afterwards. In 2017, our group pushed the resolutions of porcine and human $S \mathrm{SCl}_{1} I_{2} \mathrm{IV}_{1}$ to $4.0 \AA$ and $3.9 \AA$ (Guo et al., 2017). Our firstly obtained human respirasome structure provided the most accurate and detailed map of mutations related to severe mitochondrial malfunction diseases, including Alzheimer's disease, Huntington's disease, Parkinson's disease, Friedreich's ataxia and so on. This map of mutations could help drug-development and provide reference for prenatal diagnose of inheritable genetic diseases.

Until the year 2019, structures of mammalian $S C_{1} \|_{2} I_{1}$ in different states from various species have been deposited into PDB, laying the foundation for further discussion of the functional mechanism (Fig. $1 \mathrm{~A}$ and $1 \mathrm{~B}$ ). The mammalian respiratory $\mathrm{SCl}_{1} \mathrm{II}_{2} \mathrm{IV}_{1}$ has a dimension of around $300 \AA$ in length and $190 \AA$ in height. The L-shaped $\mathrm{Cl}$ is composed of a transmembrane arm and a hydrophilic arm (matrix arm). The TM arm is located in the mitochondrial IM, bending slightly inward with its concave surface interacting with $\mathrm{CII}_{2}$. CIV is positioned on the concave surface formed by $\mathrm{CII}_{2}$ and the distal end of the TM arm of $\mathrm{Cl}$. According to our structures, the final human $\mathrm{SCl}_{1} \mathrm{II}_{2} \mathrm{IV}_{1}$ contains 45 subunits from $\mathrm{Cl}, 21$ subunits from $\mathrm{CIII}$ and 14 subunits from CIV, and possesses 133 transmembrane helices (TMHs), with 78, 26 and $29 \mathrm{TMH}$ from $\mathrm{Cl}, \mathrm{CIII}$ and CIV, respectively.

The interactions between $\mathrm{Cl}$ and $\mathrm{Clll}$ in mammalian $\mathrm{SCl}_{1} \mathrm{II}_{2} \mathrm{IV}_{1}$ mainly take place in two regions: the first where the NDUFA11 and NDUFB4 subunits of $\mathrm{Cl}$ interact with UQCRQ subunit of CIII, and the second where the NDUFB9 and NDUFB4 subunits of $\mathrm{Cl}$ interact with the UQCRC1, UQCRFS1 subunits of CIII (Gu et al., 2016; Letts et al., 2016; Sousa et al., 2016; Wu et al., 2016; Guo et al., 2017; Milenkovic et al., 2017; Hirst, 2018).

The cytochrome c oxidase subunit VIla polypeptide 2-like protein (Cox7A2L), also known as supercomplex assembly factor 1 (SCAF1), is proposed to be essential for the interaction between CIII and CIV but does not have significant impact on the assembly or function of the respirasome (Ikeda et al., 2013; Lapuente-Brun et al., 2013; Mourier et al., 2014; Perez-Perez et al., 2016). The Cox7A2 subunit in our human CIV structure may consist with results reported in mouse strain C57BL/6 that mutation of its long isoform Cox7A2L impairs the formation of the $S C I_{2} I_{1}$, while respirasomes can exists in fully assembled form in mice expressing both long and short forms of Cox7A2L (Mourier et al., 2014; Cogliati et al., 2016; Williams et al., 2016). Previous research also indicate that Cox7A1/A2 and Cox6A1/A2 are tissue-specific expressed subunits, with Cox7A1 and Cox6A2 promoting CIV dimerization (Cogliati et al., 2016; Milenkovic et al., 2017). The Cox7A1 and Cox6A2 subunits in our monomeric human CIV structure are replaced by Cox7A2 and Cox6A1 subunits, which is consistent with the previous conclusion (Zong et al., 2018b).

Phospholipids play an important role in maintaining the structure and function of respirasomes. The formation, stabilization and function of respirasomes are impacted by the lipid composition of the IMM. The most discussed phospholipids include phosphatidylcholine (PC), phosphatidylethanolamine (PE), and cardiolipin (CDL). The phospholipids identified in the published structures of respirasomes confirmed their importance in stabilizing respirasomes and helping respirasomes complete conformational change and fulfill their functions (Zhang et al., 2002; Enríquez, 2016; Letts et al., 2016; Wu et al., 2016; Guo et al., 2017; Milenkovic et al., 2017) (Fig. 1C).

The mechanism underlying the conformational change between "active" and "deactive" states of $\mathrm{Cl}$ has been discussed following the reporting of $\mathrm{Cl}$ structures (Fiedorczuk et al., 2016; Agip et al., 2018; Blaza et al., 2018; Parey et al., 2018). The conserved features of $\mathrm{Cl}$ in different species including $\pi$-bulges, interrupted $\mathrm{TMHs}$ and charged residues in the membrane plane could form the structural basis for the function of $\mathrm{Cl}$. The deactivation of $\mathrm{Cl}$ may be related to the structural disorder in the $\mathrm{Q}$ binding site and the conformational change of the TMH3 in ND6 (Agip et al., 2019; Letts et al., 2019).

\section{AND CIV, NEWS IN OLDS}

Mammalian $\mathrm{CII}_{2}$ has long been regarded as a homodimer consisting of 22 subunits in total in the past structural studies. But the full length $\mathrm{N}$-terminal processed peptide (UQCRFS1N) of the iron-sulfur Rieske protein (UQCRFS1) subunit was not assigned in all of these structures. By rebuilding the high-resolution crystal structures of bovine and chicken CIII and analyzing the reconstructed density map of cryo-EM, we draw a new conclusion that the two 10-subuint CIII protomers are linked by a single UQCRFS1N molecule. The $\mathrm{N}$-terminal segment and the C-terminal segment of one UQCRFS1N molecule bind with each CIII protomer respectively, with the two protomers being identical. Both protomers of $\mathrm{CIII}_{2}$ are able to bind with $\mathrm{Cl}$, and consequently our reconstructed cryo-EM density map of $\mathrm{SCl}_{1} \mathrm{II}_{2} \mathrm{IV}_{1}$ show a mixed feature of the two possibilities (Zong et al., 2018a). TTC19 protein is reported to be essential for removing UQCRFS1N from the full-length UQCRFS1 subunit. In mitochondria lacking TTC19, CIII 
contains two full-length UQCRFS1 with two UQCRFS1N segments. Experimental results showed that the native molecular mass of $\mathrm{Clll}_{2}$ from Ttc19-/- knockout mouse is slightly higher than that of $\mathrm{ClI}_{2}$ from wild type animals, which may indicate it to be consistent with our conclusion (Bottani et al., 2017).

NDUFA4 was originally considered as a subunit of $\mathrm{Cl}$, and although there was still controversy (Kadenbach, 2017), further studies indicated that this protein should belong to CIV (Balsa et al., 2012; Pitceathly and Taanman, 2018). However, NDUFA4 was never found in current CIV crystal structures that present as homodimers. In $\mathrm{SCl}_{1} \mathrm{III}_{2} \mathrm{IV}_{1}, \mathrm{CIV}$ rolls around the tip of $\mathrm{Cl}$. The state when CIV is relatively closer to $\mathrm{Cl}$ is more stable than the state when CIV is approaching CIII, and the contents of particles at these two states are basically the same. This means that if we mix up all kinds of particles, the auto-refine of complex IV by adding a soft mask would fail due to the excessive differences between these two parts. If we only use one conformation, the resolution of the $S \mathrm{SC}_{1} \mathrm{III}_{2} \mathrm{IV}_{1}$ would be too low to provide a sufficient accuracy for local optimization. Therefore, we put all particles together and refine it to the highest resolution. After adding a soft mask to CIV, we classify the particles into different conformations without performing particle alignment. In the last step, the class with clear structure and the highest proportion of particles was chosen and refined. Eventually the reconstructed density map of human CIV at a resolution of $3.3 \AA$ was obtained, where we found the precise location of NDUFA4 subunit.

NDUFA4 may hamper the formation of CIV dimer by binding to the dimeric interface of the crystal structures of CIV. The TMH of NDUFA4 in CIV monomer clash with that of Cox6A2 in another protomer of CIV dimer. BN-PAGE analysis in different species with different detergents and 2D crystal data in lipid bilayer indicate that CIVs may exist as monomers in membranes, while NDUFA4 subunit is important for the formation of CIV (Wittig et al., 2006a; Balsa et al., 2012; Osuda et al., 2016). We concluded that NDUFA4 is a subunit, not an assembly factor, of CIV, and CIVs are more likely to exist as monomers in native state.

\section{FIND A WAY IN THE MAZE FOR ELECTRONS}

One of the most important goals for in studying the structure of ETC complexes and supercomplexes is to understand the functional mechanism of the respiratory chain. Respirasome was supposed to enhance the electron transporting efficiency through substrate channeling (Vartak et al., 2013; Genova and Lenaz, 2014; Lobo-Jarne and Ugalde, 2018). The interaction between $\mathrm{Cl}$ and CIII may contribute to the functional division of Q pool (Heron et al., 1978; Ragan and Heron, 1978; Schägger and Pfeiffer, 2000; Bianchi et al., 2004) and is also conducive to the modulation of ROS generation by isolating reactive intermediates (Wang et al., 2010; Maranzana et al., 2013; Letts et al., 2016). Assembly into supercomplexes also conduces to the stabilization and activation of Cl (Lamantea et al., 2002; Acin-Perez et al., 2004; Schagger et al., 2004; Vempati et al., 2009).

Biochemical experimental results have indicated the functionally relevant association between $\mathrm{Cl}$ and $\mathrm{CIII}$, and the CIII-CIV interaction also induces the possibility of a cyt $\mathrm{c}$ pool. Therefore, a widely held opinion regards the enhanced catalysis of the respirasome to be achieved by the substrate channeling translocating intermediate substrates from complex to complex and the assembly of ETC complexes to be the result of the functional segmentation of $Q$ pools and cyt $c$ pools (Genova and Lenaz, 2011; Enríquez, 2016; Hirst, 2018).

Evidence have been provided against the existence of a single $Q$ pool (Lenaz and Genova, 2012). Further spectroscopic and kinetic analysis also show the existing contradictions with both the proposals of a single or two fully independent pools ( $Q_{N A D H}$ pool and $Q_{F A D}$ pool) (Blaza et al., 2014). Recent studies pointed out that the $\mathrm{QH}_{2}$ from $\mathrm{Cl}$ is reoxidized more rapidly by added AOX outside the SC than by $\mathrm{CIII}$ inside the SC, strongly questioning the existence of the substrate channel and the segmented Q pool (Milenkovic et al., 2017; Fedor and Hirst, 2018; Hirst, 2018). Meanwhile, no confining protein structures are found between $\mathrm{Cl}$ and $\mathrm{CIII}$ that could guide the diffusion of $\mathrm{Q} / \mathrm{QH}_{2}$ (Gu et al., 2016; Letts et al., 2016; Sousa et al., 2016; Wu et al., 2016; Guo et al., 2017). Scientists also suggest that a strictly restrained channel and $Q$ pool may increase the risk of the system resisting the dysfunction of ETC complexes (Hirst, 2018). Additionally, the existence of functionally segmented cyt $\mathrm{c}$ pool is also questioned (Trouillard et al., 2011).

The prevailing view of the electron transfer process in CIII is described by $Q$ cycle theory first proposed by Peter Mitchell (Mitchell, 1975a, b; Crofts et al., 1983). This theory indicates that the oxidation of $\mathrm{QH}_{2}$ at the $\mathrm{Q}_{\mathrm{o}}$ site is a bifurcated reaction. In the reaction, $\mathrm{QH}_{2}$ binds to $\mathrm{Q}_{\mathrm{o}}$ site, while $\mathrm{Q}$ binds to $Q_{i}$ site. Two electrons obtained from the oxidation of $\mathrm{QH}_{2}$ flow to ISP and heme $\mathrm{b}_{\mathrm{L}}$, respectively. ISP accept one electron and pass it on to cytochrome $c_{1}$, then to cyt c. The other electron transports along the chain heme $b_{\mathrm{L}}$-heme $b_{\mathrm{H}^{-}}$ $Q$ (bound to $Q_{i}$ site). Two cycles are needed to reduce the $Q$ bound at $Q_{i}$ site to $\mathrm{QH}_{2}$ (Pietras et al., 2016; Sousa et al., 2018) (Fig. 3A).

Despite that the $Q$ cycle theory has been popular for a long time, there is still no high-resolution structure or reliable data to support the claim that the $\mathrm{Q}_{0}$ site is a functional $\mathrm{QH}_{2^{-}}$ binding site (Pietras et al., 2016). The difficulty in identification of the intermediate states of $Q_{0}$ site catalysis, as well as the necessity of avoiding semiquinone- mediated short circuits has also left a high degree of freedom for mechanistic interpretation (Osyczka et al., 2004; Osyczka et al., 2005; Pietras et al., 2016).

After analyzing the structure of CIII, we propose a new mechanism for the electron transfer process of CIII. In our model, $\mathrm{QH}_{2}$ released from $\mathrm{Cl}$ binds to $\mathrm{Q}_{\mathrm{i}}$ site of CIII. Heme $b_{H}$ accepts one electron from $\mathrm{QH}_{2}$ and passes it to heme $\mathrm{b}_{\mathrm{L}}$, while accepting the other electron simultaneously. The 
A

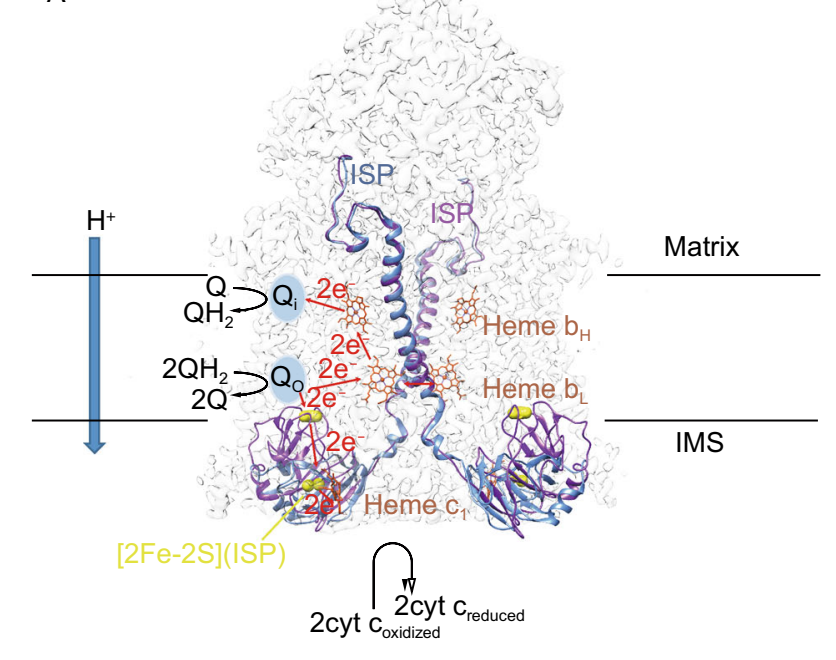

B

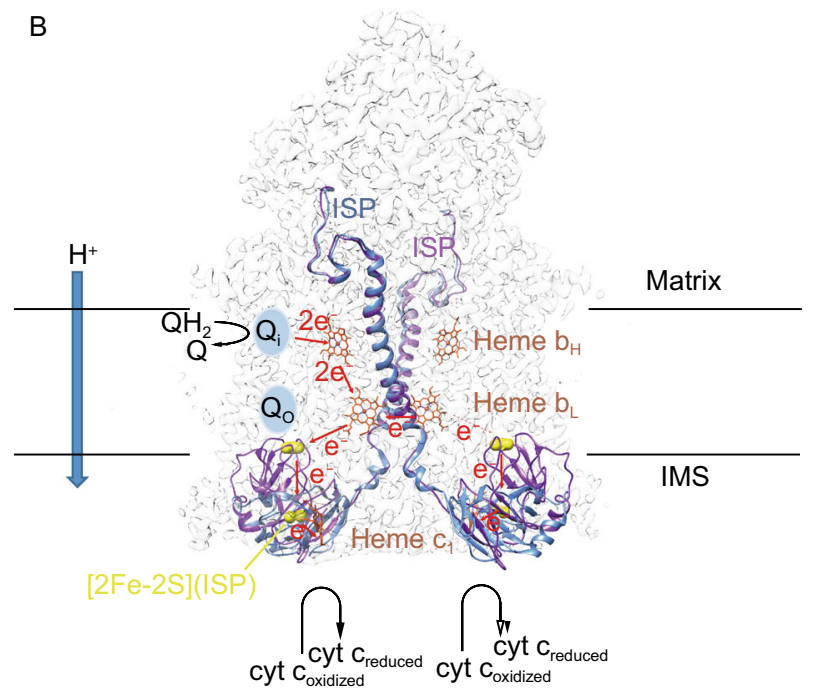

Figure 3. Mechanisms of $\mathrm{CIII}_{2}$. (A) Diagram of $\mathrm{Q}$ cycle theory. The ISP proteins and catalytic centers are shown in different colors. The ISP subunit colored in blue (PDB ID: 5 XTE, also called as " $\mathrm{c}_{1}$ state") and the ISP subunit colored in purple (PDB ID: $3 \mathrm{H} 1 \mathrm{I}$, also called as "b state") (Zhang et al., 1998) indicate the conformational change between two states of CIII during electron transportation. The black dotted lines represent the mitochondrial inner membrane. The black arrows represent the change of substrates. The blue arrow indicates the proton translocation direction. The red dotted lines indicates the electron flow in CIII dimer. The light blue circles represent the $Q$-binding site $Q_{i}$ and $Q_{0}$. (B) Diagram of the mechanism raised in our paper. The labels are consist with Fig. 3A.

oxidized $Q$ is released from $Q_{i}$ site. Heme $b_{L}$ accepts the first electron and transfers it to the heme $b_{L}$ in the other CIII monomer, enabling the first heme $b_{L}$ to accept the other electron from heme $b_{H}$. Heme $b_{L}$ transfers the electrons to ISP, and then to cyt $\mathrm{c}_{1}$, and eventually cyt $\mathrm{c}$. In this way, the accumulation of semi-quinone radical is reduced, thus minimizing the generation of ROS. At the same time, as the electron transportation between two heme $b_{L}$ molecules could be faster than the conformational change of ISP, the electron transfer between heme $b_{L}$ molecules could occur earlier in our model (Wu et al., 2016; Guo et al., 2017) (Fig. 3B). During this process, two protons dissociated from $\mathrm{QH}_{2}$ are translocated to the IMS using the redox energy released by electron transfer.

Our model provides an explanation to the phenomenon that the addition of myxothiazol, an inhibitor bound at $Q_{0}$ site, could stimulate generation of superoxide at $Q_{0}$ site (Starkov and Fiskum, 2001; Muller et al., 2002). After two electrons are transferred from $\mathrm{QH}_{2}$ at $\mathrm{Q}_{\mathrm{i}}$ site to two heme $\mathrm{b}_{\mathrm{L}}$, conformational change of ISP becomes the rate limiting step. The binding of myxothiazol even further slow down the conformational change of ISP, causing electrons backing up at heme $b_{L}$, becoming the source of superoxide.

The asymmetric $Q$ binding densities in CIII segment of $\mathrm{SCl}_{1} \mathrm{Ill}_{2}$ may indicate a more complicated functional mechanism (Letts et al., 2019). More research are needed to clarify the mechanism of electron transportation in individual ETC complexes and the respirasome as a whole, which could include analyzing atomic structures of SCs at more states and conducting related biophysical and biochemical experiments. It is possible that EPR could help us understand the pathway of electron transfer in the respirasome (Ohnishi et al., 2012; Pietras et al., 2016; Wright et al., 2016).

\section{MEGACOMPLEX, CALLING FOR TECHNOLOGY PROGRESS}

The concept of megacomplex has long been brought along by EM/ET analyses. In the early studies, the possibility of the existence of dimeric $\mathrm{Cl}$ was proposed, accompanied by the anticipation of finding convincing evidence for a respiratory string composed of $\mathrm{Cl}, \mathrm{CIII}$ and CIV (Allen et al., 1989; Nubel et al., 2009; Wittig and Schagger, 2009; Strecker et al., 2010). Accordingly, models of respiratory strings with different basic units and conjunction modes were raised (Wittig et al., 2006b; Bultema et al., 2009; Vonck, 2012; Letts et al., 2016). $\mathrm{MCl}_{2} \mathrm{II}_{2} I \mathrm{~V}_{2}$ was first supposed to be the block of a modality of respiratory string in our results published in 2016 (Wu et al., 2016), inspired by the identification of the supramolecular assembly form $\mathrm{I}_{2} \mathrm{III}_{2}$ in potato (Bultema et al., 2009).

During our study of the respirasome, we noticed the existence of higher-order assemblies of ETC complexes as have been indicated by previous investigations. First, we detected high-molecular-weight bands above $\mathrm{SCl}_{1} \mathrm{III}_{2} \mathrm{IV} \mathrm{V}_{1}$ in BN-PAGE analysis. The results of mass spectrometry analysis suggest that the main components of these bands are subunits of the ETC complexes, particularly $\mathrm{CI}, \mathrm{CIII}$ and CIV. 
Negative stained samples were prepared for EM examination. A minor population of particles with an circular arrangement were detected. These findings led to our proposal of a higher oligomeric state named megacomplex$\mathrm{I}_{2} \mathrm{II}_{2} \mathrm{IV}_{2}\left(\mathrm{MCl}_{2} \mathrm{II}_{2} \mathrm{IV}_{2}\right)$ published in 2016. We assumed this assembly form as a structure with dimeric CIII located in the center, surrounded by two $\mathrm{Cls}$ with their membrane arms oriented perpendicularly to the 2-fold axis of $\mathrm{CIII}_{2}$. Two CIVs were anchored by the distal end of each $\mathrm{Cl}$ membrane arm and $\mathrm{CII}_{2}$. The $\mathrm{MCl}_{2} \mathrm{III}_{2} \mathrm{IV}_{2}$ contains 139 subunits in total (45 from each $\mathrm{Cl}, 14$ from each $\mathrm{CIV}$, and 21 from $\mathrm{CIII}_{2}$ ), with a height of $\sim 220 \AA$, width of $\sim 280 \AA$, and length of $\sim 300 \AA$ (Guo et al., 2017).

Through further analysis of large amounts of cryo-EM data, we obtained the $3 \mathrm{D}$ reconstruction architecture of $\mathrm{MCl}_{2} \mathrm{III}_{2} \mathrm{IV}_{2}$ at a resolution of $17.4 \AA$ in 2017 with about 8,600 particles out of 1.18 million (Guo et al., 2017) (Fig. 1D). These results provoked intense debates immediately. Skeptical opinions center around the point that $\mathrm{MCl}_{2} \mathrm{II}_{2} \mathrm{IV}_{2}$ might be an artifact caused by the usage of specific detergent (digitonin) or the tendentious collection of nonspecific particles, because of the extremely low relative content and the lack of in situ detection or biochemical experimental evidence. However, NBT staining of our BN-PAGE result indeed detected a catalytically active band above the band of respirasome (Guo et al., 2017), which we believe to be the proof of existing higher organization form than respirasome.

In our following study, we improved the relative concentration of $\mathrm{MCl}_{2} \mathrm{II}_{2} \mathrm{IV}_{2}$ and pushed the reconstruction results to a better resolution where secondary structures can be clearly visualized. Different initial models and unsupervised ab initio reconstructions are tried to avoid model-bias effect. $\mathrm{MCl}_{2} \mathrm{II}_{2} \mathrm{IV}_{2}$ are found both in porcine and human derived mitochondrial extracts. According to our megacomplex structure with higher resolution, we can even detect direct interactions between CIII and two Cls, and two CIVs bind to CIII in a different pattern from respirasome (unpublished data). However, these progresses did not dispel all the suspicion, mostly because no megacomplex was resolved from cryo-ET images. We must admit the relative ratio of $\mathrm{MCl}_{2} \mathrm{III}_{2} \mathrm{IV}_{2}$ is quite low, so it could be difficult to detect $\mathrm{MCl}_{2} \mathrm{III}_{2} \mathrm{IV}_{2}$ from a multiple of mitochondrial slices. In contrast, during single particle data processing, proteins from mitochondria in various states can be extracted, so the chance of detecting $\mathrm{MCl}_{2} \mathrm{II}_{2} \mathrm{IV}_{2}$ is appearently higher. Therefore, technology progress of cryo-ET is in urgent need to reconstruct supramolecular assemblies in mitochondria extracted from different stages of cell division cycle.

Cryo-ET and subtomogram averaging results of mitochondrial membranes strengthened the skepticism about the existence of the ubiquitous respiratory strings, suggesting that $\mathrm{SCl}_{1} \mathrm{II}_{2}$ is the most conserved structure across different species (Davies et al., 2011; Davies et al., 2018). In contrast, however, the recently published structure of $S C \mathrm{III}_{2} \mathrm{IV}_{1-2}$ from Mycobacterium smegmatis and yeast may indicate the functional necessity of a cytochrome subunit bridged electron transfer path for ETC complexes in prokaryotic cells (Gong et al., 2018; Wiseman et al., 2018; Hartley et al., 2019; Rathore et al., 2019).

As a powerful tool for in situ reconstruction, cryo-ET is one of the most promising technology that might be able to extricate us from the current predicament. However, cryo-ET is facing the dilemma once faced by cryo-EM single particle analysis - the bottleneck of limited resolution, which is a comprehensive result of manifold causes. For cryo-ET, effective methods still need to be derived in areas including sample preparation, determination of the area to be detected, phase plate performance, motion correction and tilt series alignment, CTF estimation, treatment for the loss of information at high tilt angles, image processing algorithm and many more. Subtomogram averaging provides a scheme to balance the advantages of both single particle analysis and traditional cryo-tomography methods (Wan and Briggs, 2016; Leigh et al., 2019; Schur, 2019). With the application of newly developed 3D-CTF estimation algorithm, the resolution of subtomogram averaging reconstruction could reach $3.4 \AA$ (Turonova et al., 2017). This methodology is largely a variant of single particle analysis, thus the large-scale in situ reconstruction at high resolution still needs the invention of new techniques and new ideas.

Time-resolved cryo-EM is also a possible developing trend depending on better classification methods and dynamic simulation algorithms (White et al., 2003; Fu et al., 2016; Frank, 2017b). As the star technology gaining great attention, cryo-EM/ET still has tremendous potential to be explored.

\section{CONCLUSIONS AND PERSPECTIVES}

The curiosity into respiration encapsulates people's desire for exploring the mysteries of life. Through the efforts of scientists over the past century, features of the molecular machineries that are responsible for this physiological process have become gradually known to us. The rapid development in cryo-EM technology provides us with a powerful tool to break through the bottleneck of analyzing the atomic structure of macromolecular protein complexes, pushing the research field of respirasome forward into a new stage. Highresolution structures of eukaryotic respiratory supercomplex $\mathrm{I}_{1} \mathrm{II}_{2} \mathrm{IV} \mathrm{V}_{1}$ and $\mathrm{III}_{2} \mathrm{IV} \mathrm{V}_{2}$ have been acquired successively. New features of mammalian ETC complex III and IV have also come into view. The discovery of $\mathrm{MCl}_{2} \mathrm{II}_{2} \mathrm{IV}$ may provide a new possibility to the study of the electron transfer mechanism. With new theoretical arguments and experimental results emerging, widely accepted theories are starting to be challenged. All of these results together could help us clarify the functional mechanism of the respirasome, which is of great significance to elucidating the pathogenesis of mitochondrial diseases and finding the treatment methods of mitochondrial diseases.

ETCs are the most abundant protein within mitochondrial inner membrane, and they are closely related to the 

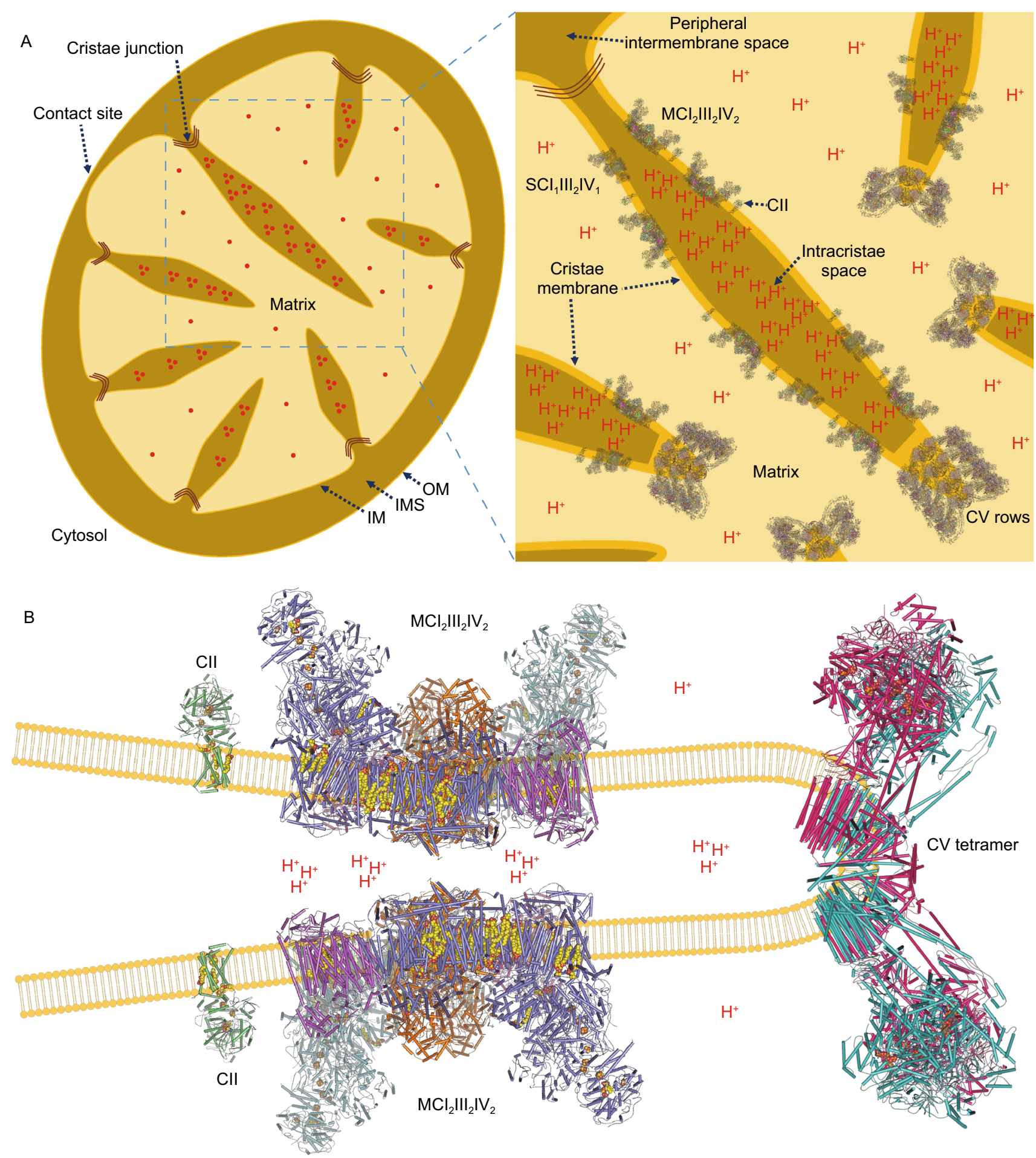

Figure 4. Orgnization and distribution of mammalian ETC complexes in mitochondria. (A) Diagram of mitochondrial internal morphology (left). The part in the blue dotted frame is enlarged (right) with the simulated organization and distribution of ETC complexes shown. The proton concentration gradient is represented with red notes. PDB ID of CII: $1 Z O Y$. PDB ID of $\mathrm{MCl}_{2} I_{2} \mathrm{IV}_{2}$ : 5XTI. PDB ID of $\mathrm{SCl}_{1} \mathrm{III}_{2} \mathrm{IV} \mathrm{V}_{1}$ : $5 \mathrm{XTH}$. PDB ID of CV: $6 \mathrm{~J} 5 \mathrm{~K}$. (B) Distribution of Cll, $\mathrm{MCl}_{2} \mathrm{Ill}_{2} \mathrm{IV} \mathrm{V}_{2}$ and $\mathrm{CV}$ tetramer on mitochondrial cristae. Cofactors are shown in spheres. The structures used are the same with Fig. $4 \mathrm{~A}$. 
maintaining of cristae shape. Taken our structures and the in situ cryo-ET images together, it is now widely accepted that the CV oligomers sit on the tip of cristae ridges, while respiratory chain complexes are located on the relatively flat cristae plane, whether assemble into supercomplex (even megacomplex) or stay alone. Protons are enriched in the intracristae space, especially at the cristae tips. This arrangement of ETCs could be most efficient in energy conversion (Fig. 4A and 4B), For complexes I, II, III, IV, and V in bovine heart mitochondria, a ratio $(1.1 \pm 0.2):(1.3 \pm 0.1): 3:(6.7 \pm 0.8):(3.5 \pm 0.2)$ was determined (Schagger and Pfeiffer, 2001). Appearantly, the ratio of CIV is much higher than $\mathrm{Cl}$ and $\mathrm{CIII}$, so the binding pattern of CIV could be either with $\mathrm{CI}$, CIII, or staying alone. Actually, we even identified some respirasome particles containing more than one copy of CIV.

However, the recent debates over the new proposals indicate that much more evidence are still needed to dispel the doubts. In situ reconstruction of respirasomes under different cell conditions might provide a break point to the current situation. In situ and time- resolution reconstruction are the developing directions of cryo-EM/ET that have received wide attention. For cryo-ET, resolution of the $3 D$ reconstruction is still the most prominent limitation for its application. The advances in super-resolution light microscopy may also be useful for dynamic research of respirasomes. In addition, more biochemical and biophysical experiments are also needed to help clarify the speculations.

The history for scientists to get to know respirasome is a combination of technological development and theoretical innovation, which also holds true in the broad scientific world. With the combined effort of all scientists in this field, we expect that one day the whole picture of respiration could be presented to us.

\section{ABBREVIATIONS}

ATP, adenosine triphosphate; BN-PAGE, blue native polyacrylamide gel electrophoresis; CDL, cardiolipin; CCD, charge-coupled devices; CI, complex I; CII, complex II; CIII, complex III; CIV, complex IV; CV, complex V; cryo-EM, cryo-electron microscopy; cyt b, cytochrome b; cyt c, cytochrome c; cyt c1, cytochrome c1; DQE, detective quantum efficiency; DDD, direct electron detection devices; ETC, electron transport chain; FMN, flavin mononucleotide; IMM, inner mitochondrial membrane; IMS, intermembrane space; ISP, iron-sulfur protein; MC, megacomplex; MM, mitochondrial matrix; NMR, nuclear magnetic resonance; OXPHOS, oxidative phosphorylation; $\mathrm{PC}$, phosphatidylcholin; PE, phosphatidylethanolamin; PDB, Protein Data Bank; PMF, proton-motive force; ROS, reactive oxygen species; SC, supercomplexe; TMH, transmembrane helices; $\mathrm{QH} 2$, ubiquinol-10; $\mathrm{Q}$, ubiquinone-10

\section{ACKNOWLEDGEMENTS}

We thank the Tsinghua University Branch of China National Center for Protein Sciences (Beijing) for providing the facility support.

\section{COMPLIANCE WITH ETHICS GUIDELINES}

Maojun Yang, Meng Wu, Jinke Gu, Shuai Zong, Runyu Guo, and Tianya Liu declare that they have no conflict of interest.

\section{OPEN ACCESS}

This article is licensed under a Creative Commons Attribution 4.0 International License, which permits use, sharing, adaptation, distribution and reproduction in any medium or format, as long as you give appropriate credit to the original author(s) and the source, provide a link to the Creative Commons licence, and indicate if changes were made. The images or other third party material in this article are included in the article's Creative Commons licence, unless indicated otherwise in a credit line to the material. If material is not included in the article's Creative Commons licence and your intended use is not permitted by statutory regulation or exceeds the permitted use, you will need to obtain permission directly from the copyright holder. To view a copy of this licence, visit https:// creativecommons.org/licenses/by/4.0/.

\section{REFERENCES}

Acin-Perez R, Bayona-Bafaluy MP, Fernandez-Silva P, MorenoLoshuertos R, Perez-Martos A, Bruno C, Moraes CT, Enriquez JA (2004) Respiratory complex III is required to maintain complex I in mammalian mitochondria. Mol Cell 13:805-815

Acin-Perez R, Fernandez-Silva P, Peleato ML, Perez-Martos A, Enriquez JA (2008) Respiratory active mitochondrial supercomplexes. Mol Cell 32:529-539

Adiga PS, Malladi R, Baxter W, Glaeser RM (2004) A binary segmentation approach for boxing ribosome particles in cryo EM micrographs. J Struct Biol 145:142-151

Adrian M, Dubochet J, Lepault J, McDowall AW (1984) Cryo-electron microscopy of viruses. Nature 308:32-36

Agip A-NA, Blaza JN, Bridges HR, Viscomi C, Rawson S, Muench SP, Hirst J (2018) Cryo-EM structures of complex I from mouse heart mitochondria in two biochemically defined states. Nat Struct Mol Biol 25:548-556

Agip AA, Blaza JN, Fedor JG, Hirst J (2019) Mammalian respiratory complex I through the lens of cryo-EM. Annu Rev Biophys 48:165-184

Al-Azzawi A, Ouadou A, Tanner JJ, Cheng J (2019a) AutoCryoPicker: an unsupervised learning approach for fully automated single particle picking in Cryo-EM images. BMC Bioinform 20:326

Al-Azzawi A, Ouadou A, Tanner JJ, Cheng J (2019b) A superclustering approach for fully automated single particle picking in Cryo-EM. Genes (Basel). https://doi.org/10.3390/ genes 10090666

Alcázar-Fabra M, Navas P, Brea-Calvo G (2016) Coenzyme Q biosynthesis and its role in the respiratory chain structure. Biochim Biophys Acta (BBA) 1857:1073-1078

Allen RD, Schroeder CC, Fok AK (1989) An investigation of mitochondrial inner membranes by rapid-freeze deep-etch techniques. J Cell Biol 108:2233-2240 
Althoff T, Mills DJ, Popot JL, Kuhlbrandt W (2011) Arrangement of electron transport chain components in bovine mitochondrial supercomplex I1III2IV1. EMBO J 30:4652-4664

Bai XC, Fernandez IS, McMullan G, Scheres SH (2013) Ribosome structures to near-atomic resolution from thirty thousand cryo-EM particles. Elife 2:e00461

Bai XC, McMullan G, Scheres SH (2015) How cryo-EM is revolutionizing structural biology. Trends Biochem Sci 40:49-57

Balsa E, Marco R, Perales-Clemente E, Szklarczyk R, Calvo E, Landazuri MO, Enriquez JA (2012) NDUFA4 is a subunit of complex IV of the mammalian electron transport chain. Cell Metab 16:378-386

Barquera B (2014) The sodium pumping NADH:quinone oxidoreductase ( $\mathrm{Na}(+)-\mathrm{NQR})$, a unique redox-driven ion pump. J Bioenerg Biomembr 46:289-298

Bates MGD, Bourke JP, Giordano C, d'Amati G, Turnbull DM, Taylor RW (2012) Cardiac involvement in mitochondrial DNA disease: clinical spectrum, diagnosis, and management. Eur Heart $\mathrm{J}$ 33:3023-3033

Bentinger M, Tekle M, Dallner G (2010) Coenzyme Q_biosynthesis and functions. Biochem Biophys Res Commun 396:74-79

Bezawork-Geleta A, Rohlena J, Dong L, Pacak K, Neuzil J (2017) Mitochondrial complex II: at the crossroads. Trends Biochem Sci 42:312-325

Biagini GA, Fisher N, Shone AE, Mubaraki MA, Srivastava A, Hill A, Antoine T, Warman AJ, Davies J, Pidathala C et al (2012) Generation of quinolone antimalarials targeting the Plasmodium falciparum mitochondrial respiratory chain for the treatment and prophylaxis of malaria. Proc Natl Acad Sci USA 109:8298-8303

Bianchi C, Genova ML, Parenti Castelli G, Lenaz G (2004) The mitochondrial respiratory chain is partially organized in a supercomplex assembly: kinetic evidence using flux control analysis. J Biol Chem 279:36562-36569

Blair PV (1967) Preparation and properties of repeating units of mitochondrial electron transfer. Methods Enzymol 10:208-212

Blaza JN, Serreli R, Jones AJ, Mohammed K, Hirst J (2014) Kinetic evidence against partitioning of the ubiquinone pool and the catalytic relevance of respiratory-chain supercomplexes. Proc Natl Acad Sci USA 111:15735-15740

Blaza JN, Vinothkumar KR, Hirst J (2018) Structure of the deactive state of mammalian respiratory complex I. Structure 26:312-319. e313

Bottani E, Cerutti R, Harbour ME, Ravaglia S, Dogan SA, Giordano C, Fearnley IM, D’Amati G, Viscomi C, Fernandez-Vizarra E et al (2017) TTC19 plays a husbandry role on UQCRFS1 turnover in the biogenesis of mitochondrial respiratory complex III. Mol Cell 67:96-105.e104

Brandt U (2006) Energy converting NADH:quinone oxidoreductase (complex I). Annu Rev Biochem 75:69-92

Brandt U (2011) A two-state stabilization-change mechanism for proton-pumping complex I. Biochim Biophys Acta 1807:13641369

Brilot AF, Chen JZ, Cheng A, Pan J, Harrison SC, Potter CS, Carragher B, Henderson R, Grigorieff N (2012) Beam-induced motion of vitrified specimen on holey carbon film. J Struct Biol 177:630-637
Brzezinski P, Adelroth P (1998) Pathways of proton transfer in cytochrome c oxidase. J Bioenerg Biomembr 30:99-107

Bultema JB, Braun HP, Boekema EJ, Kouril R (2009) Megacomplex organization of the oxidative phosphorylation system by structural analysis of respiratory supercomplexes from potato. Biochim Biophys Acta 1787:60-67

Cecchini G (2003) Function and structure of complex II of the respiratory chain. Annu Rev Biochem 72:77-109

Chance B, Estabrook RW, Lee CP (1963) Electron transport in the oxysome. Science 140:379-380

Chance B, Williams GR (1955) A method for the localization of sites for oxidative phosphorylation. Nature 176:250

Cheng Y (2015) Single-particle cryo-EM at crystallographic resolution. Cell 161:450-457

Cogliati S, Calvo E, Loureiro M, Guaras AM, Nieto-Arellano R, Garcia-Poyatos C, Ezkurdia I, Mercader N, Vazquez J, Enriquez JA (2016) Mechanism of super-assembly of respiratory complexes III and IV. Nature 539:579-582

Crane FL, Hatefi Y, Lester RL, Widmer C (1957) Isolation of a quinone from beef heart mitochondria. Biochim Biophys Acta 25:220-221

Crofts AR, Meinhardt SW, Jones KR, Snozzi M (1983) The role of the quinone pool in the cyclic electron-transfer chain of rhodopseudomonas sphaeroides: a modified Q-cycle mechanism. Biochim Biophys Acta 723:202-218

Davies KM, Blum TB, Kuhlbrandt W (2018) Conserved in situ arrangement of complex I and III2 in mitochondrial respiratory chain supercomplexes of mammals, yeast, and plants. Proc Natl Acad Sci USA 115:3024-3029

Davies KM, Strauss M, Daum B, Kief JH, Osiewacz HD, Rycovska A, Zickermann V, Kuhlbrandt W (2011) Macromolecular organization of ATP synthase and complex I in whole mitochondria. Proc Natl Acad Sci USA 108:14121-14126

De Rosier DJ, Klug A (1968) Reconstruction of three dimensional structures from electron micrographs. Nature 217:130-134

Dubochet J, Adrian M, Chang JJ, Homo JC, Lepault J, McDowall AW, Schultz P (1988) Cryo-electron microscopy of vitrified specimens. Q Rev Biophys 21:129-228

Dubochet J, Booy FP, Freeman R, Jones AV, Walter CA (1981) Low temperature electron microscopy. Annu Rev Biophys Bioeng 10:133-149

Duchen MR, Szabadkai G (2010) Roles of mitochondria in human disease. Essays Biochem 47:115-137

Dudkina NV, Kudryashev M, Stahlberg H, Boekema EJ (2011) Interaction of complexes I, III, and IV within the bovine respirasome by single particle cryoelectron tomography. Proc Natl Acad Sci USA 108:15196-15200

Efremov RG, Sazanov LA (2012) The coupling mechanism of respiratory complex I - a structural and evolutionary perspective. Biochim Biophys Acta 1817:1785-1795

Enríquez JA (2016) Supramolecular organization of respiratory complexes. Annu Rev Physiol 78:533-561

Eubel H, Heinemeyer J, Sunderhaus S, Braun HP (2004) Respiratory chain supercomplexes in plant mitochondria. Plant Physiol Biochem 42:937-942

Evans DR, Guy HI (2004) Mammalian pyrimidine biosynthesis: fresh insights into an ancient pathway. J Biol Chem 279:33035-33038 
Fan X, Wang J, Zhang X, Yang Z, Zhang JC, Zhao L, Peng HL, Lei J, Wang HW (2019) Single particle cryo-EM reconstruction of 52 $\mathrm{kDa}$ streptavidin at 3.2 Angstrom resolution. Nat Commun 10:2386

Fedor JG, Hirst J (2018) Mitochondrial supercomplexes do not enhance catalysis by quinone channeling. Cell Metab 28:525531.e524

Feng X, Fu Z, Kaledhonkar S, Jia Y, Shah B, Jin A, Liu Z, Sun M, Chen B, Grassucci RA et al (2017) A fast and effective microfluidic spraying-plunging method for high-resolution singleparticle cryo-EM. Structure 25:663-670.e663

Feng Y, Li WF, Li J, Wang JW, Ge JP, Xu D, Liu YJ, Wu KQ, Zeng QY, Wu JW et al (2012) Structural insight into the type-II mitochondrial NADH dehydrogenases. Nature 491:478-482

Fiedorczuk K, Letts JA, Degliesposti G, Kaszuba K, Skehel M, Sazanov LA (2016) Atomic structure of the entire mammalian mitochondrial complex I. Nature 538:406

Frank J (2017a) Advances in the field of single-particle cryo-electron microscopy over the last decade. Nat Protoc 12:209-212

Frank J (2017b) Time-resolved cryo-electron microscopy: Recent progress. J Struct Biol 200:303-306

Frank J, Shimkin B, Dowse H (1981) Spider-a modular software system for electron image processing. Ultramicroscopy 6:343357

Frenzel M, Rommelspacher H, Sugawa MD, Dencher NA (2010) Ageing alters the supramolecular architecture of OxPhos complexes in rat brain cortex. Exp Gerontol 45:563-572

Friedrich T (2014) On the mechanism of respiratory complex I. J Bioenerg Biomembr 46:255-268

Fu Z, Kaledhonkar S, Borg A, Sun M, Chen B, Grassucci RA, Ehrenberg M, Frank J (2016) Key intermediates in ribosome recycling visualized by time-resolved cryoelectron microscopy. Structure 24:2092-2101

Genova ML, Lenaz G (2011) New developments on the functions of coenzyme $Q$ in mitochondria. BioFactors 37:330-354

Genova ML, Lenaz G (2014) Functional role of mitochondrial respiratory supercomplexes. Biochim Biophys Acta 1837:427443

Gomez LA, Monette JS, Chavez JD, Maier CS, Hagen TM (2009) Supercomplexes of the mitochondrial electron transport chain decline in the aging rat heart. Arch Biochem Biophys 490:30-35

Gong H, Li J, Xu A, Tang Y, Ji W, Gao R, Wang S, Yu L, Tian C, Li J et al (2018) An electron transfer path connects subunits of a mycobacterial respiratory supercomplex. Science. https://doi.org/ 10.1126/science.aat8923

Green DE, Tzagoloff A (1966) The mitochondrial electron transfer chain. Arch Biochem Biophys 116:293-304

Greggio C, Jha P, Kulkarni SS, Lagarrigue S, Broskey NT, Boutant M, Wang X, Conde Alonso S, Ofori E, Auwerx J et al (2017) Enhanced respiratory chain supercomplex formation in response to exercise in human skeletal muscle. Cell Metab 25:301-311

Grigorieff N (1998) Three-dimensional structure of bovine NADH: ubiquinone oxidoreductase (complex I) at $22 \mathrm{~A}$ in ice. J Mol Biol 277:1033-1046

Gu J, Wu M, Guo R, Yan K, Lei J, Gao N, Yang M (2016) The architecture of the mammalian respirasome. Nature 537:639
Gu J, Zhang L, Zong S, Guo R, Liu T, Yi J, Wang P, Zhuo W, Yang M (2019) Cryo-EM structure of the mammalian ATP synthase tetramer bound with inhibitory protein IF1. Science 364:10681075

Guo R, Gu J, Zong S, Wu M, Yang M (2018) Structure and mechanism of mitochondrial electron transport chain. Biomed $\mathrm{J}$ 41:9-20

Guo R, Zong S, Wu M, Gu J, Yang M (2017) Architecture of human mitochondrial respiratory megacomplex I2III2IV2. Cell 170:12471257.e1212

Gupte SS, Hackenbrock CR (1988) The role of cytochrome c diffusion in mitochondrial electron transport. J Biol Chem 263:5248-5253

Hackenbrock CR (1977) Molecular organization and the fluid nature of the mitochondrial energy transducing membrane. In: Abrahamsson S, Pascher I (eds) Structure of biological membranes. Springer, Boston, MA, pp 199-234

Harding JW Jr, Pyeritz EA, Copeland ES, White HB 3rd (1975) Role of glycerol 3-phosphate dehydrogenase in glyceride metabolism. Effect of diet on enzyme activities in chicken liver. Biochemical Journal 146:223-229

Hartley AM, Lukoyanova N, Zhang Y, Cabrera-Orefice A, Arnold S, Meunier B, Pinotsis N, Marechal A (2019) Structure of yeast cytochrome c oxidase in a supercomplex with cytochrome bc1. Nat Struct Mol Biol 26:78-83

Hatefi Y (1985) The mitochondrial electron transport and oxidative phosphorylation system. Annu Rev Biochem 54:1015-1069

Hatefi Y, Haavik AG, Fowler LR, Griffiths DE (1962) Studies on the electron transfer system. XLII. Reconstitution of the electron transfer system. J Biol Chem 237:2661-2669

Hayward SB, Glaeser RM (1979) Radiation damage of purple membrane at low temperature. Ultramicroscopy 04:201-210

Henderson R, Baldwin JM, Ceska TA, Zemlin F, Beckmann E, Downing KH (1990) Model for the structure of bacteriorhodopsin based on high-resolution electron cryo-microscopy. J Mol Biol 213:899-929

Henderson R, Unwin PN (1975) Three-dimensional model of purple membrane obtained by electron microscopy. Nature 257:28-32

Heron C, Ragan Cl, Trumpower BL (1978) The interaction between mitochondrial NADH-ubiquinone oxidoreductase and ubiquinolcytochrome c oxidoreductase. Restoration of ubiquinone-pool behaviour. Biochem J 174:791-800

Hill R, Keilin D (1930) The porphyrin of component c of cytochrome and its relationship to other porphyrins. Proc R Soc Lond B Biol Sci 107:286-292

Hirst J (2013) Mitochondrial complex I. Annu Rev Biochem 82:551575

Hirst J (2018) Open questions: respiratory chain supercomplexeswhy are they there and what do they do? BMC Biol 16:111

Hochli M, Hackenbrock CR (1976) Fluidity in mitochondrial membranes: thermotropic lateral translational motion of intramembrane particles. Proc Natl Acad Sci USA 73:1636-1640

Hochli M, Hochli L, Hackenbrock CR (1985) Independent lateral diffusion of cytochrome bc1 complex and cytochrome oxidase in the mitochondrial inner membrane. Eur J Cell Biol 38:1-5 
Hofhaus G, Weiss H, Leonard K (1991) Electron microscopic analysis of the peripheral and membrane parts of mitochondrial NADH dehydrogenase (complex I). J Mol Biol 221:1027-1043

Hofmann AD, Beyer M, Krause-Buchholz U, Wobus M, Bornhauser M, Rodel G (2012) OXPHOS supercomplexes as a hallmark of the mitochondrial phenotype of adipogenic differentiated human MSCs. PLoS ONE 7:e35160

Hu M, Yu H, Gu K, Wang Z, Ruan H, Wang K, Ren S, Li B, Gan L, Xu $S$ et al (2018) A particle-filter framework for robust cryo-EM 3D reconstruction. Nat Methods 15:1083-1089

Hunte C, Zickermann V, Brandt U (2010) Functional modules and structural basis of conformational coupling in mitochondrial complex I. Science 329:448-451

Ikeda K, Shiba S, Horie-Inoue K, Shimokata K, Inoue S (2013) A stabilizing factor for mitochondrial respiratory supercomplex assembly regulates energy metabolism in muscle. Nat Commun 4:2147

Iverson TM, Luna-Chavez C, Cecchini G, Rees DC (1999) Structure of the Escherichia coli fumarate reductase respiratory complex. Science 284:1961-1966

Iwata S, Lee JW, Okada K, Lee JK, Iwata M, Rasmussen B, Link TA, Ramaswamy S, Jap BK (1998) Complete structure of the 11-subunit bovine mitochondrial cytochrome bc1 complex. Science 281:64-71

Iwata S, Ostermeier C, Ludwig B, Michel H (1995) Structure at 2.8 A resolution of cytochrome $\mathrm{C}$ oxidase from Paracoccus denitrificans. Nature 376:660-669

Kadenbach B (2017) Regulation of mammalian 13-subunit cytochrome $\mathrm{c}$ oxidase and binding of other proteins: role of NDUFA4. Trends Endocrinol Metab 28:761-770

Kalckar HM (1974) Origins of the concept oxidative phosphorylation. Mol Cell Biochem 5:55-62

Kalckar HM (1991) 50 years of biological research—from oxidative phosphorylation to energy requiring transport regulation. Annu Rev Biochem 60:1-38

Keilin D, Hartree EF (1947) Activity of the cytochrome system in heart muscle preparations. Biochem J 41:500-502

Kerscher S, Drose S, Zickermann V, Brandt U (2008) The three families of respiratory NADH dehydrogenases. Results Probl Cell Differ 45:185-222

Kerscher SJ (2000) Diversity and origin of alternative NADH: ubiquinone oxidoreductases. Biochim Biophys Acta 1459:274283

Konstantinov AA, Siletsky S, Mitchell D, Kaulen A, Gennis RB (1997) The roles of the two proton input channels in cytochrome $C$ oxidase from Rhodobacter sphaeroides probed by the effects of site-directed mutations on time-resolved electrogenic intraprotein proton transfer. Proc Natl Acad Sci USA 94:9085-9090

Krause $F$ (2006) Detection and analysis of protein-protein interactions in organellar and prokaryotic proteomes by native gel electrophoresis: (Membrane) protein complexes and supercomplexes. Electrophoresis 27:2759-2781

Krause F, Reifschneider NH, Vocke D, Seelert H, Rexroth S, Dencher NA (2004a) "Respirasome"-like supercomplexes in green leaf mitochondria of spinach. J Biol Chem 279:4836948375
Krause $F$, Scheckhuber $C Q$, Werner A, Rexroth $S$, Reifschneider $\mathrm{NH}$, Dencher NA, Osiewacz HD (2004b) Supramolecular organization of cytochrome $c$ oxidase- and alternative oxidasedependent respiratory chains in the filamentous fungus Podospora anserina. J Biol Chem 279:26453-26461

Kroger A, Klingenberg M (1973) The kinetics of the redox reactions of ubiquinone related to the electron-transport activity in the respiratory chain. Eur J Biochem 34:358-368

Kuijper M, van Hoften G, Janssen B, Geurink R, De Carlo S, Vos M, van Duinen G, van Haeringen B, Storms M (2015) FEl's direct electron detector developments: embarking on a revolution in cryo-TEM. J Struct Biol 192:179-187

Lamantea E, Carrara F, Mariotti C, Morandi L, Tiranti V, Zeviani M (2002) A novel nonsense mutation (Q352X) in the mitochondrial cytochrome $b$ gene associated with a combined deficiency of complexes I and III. Neuromuscul Disord 12:49-52

Langlois R, Pallesen J, Ash JT, Nam Ho D, Rubinstein JL, Frank J (2014) Automated particle picking for low-contrast macromolecules in cryo-electron microscopy. J Struct Biol 186:1-7

Lapuente-Brun E, Moreno-Loshuertos R, Acin-Perez R, LatorrePellicer A, Colas C, Balsa E, Perales-Clemente E, Quiros PM, Calvo E, Rodriguez-Hernandez MA et al (2013) Supercomplex assembly determines electron flux in the mitochondrial electron transport chain. Science 340:1567-1570

Lax NZ, Turnbull DM, Reeve AK (2011) Mitochondrial mutations: newly discovered players in neuronal degeneration. Neuroscientist 17:645-658

Leigh KE, Navarro PP, Scaramuzza S, Chen W, Zhang Y, CastanoDiez D, Kudryashev M (2019) Subtomogram averaging from cryo-electron tomograms. Methods Cell Biol 152:217-259

Lenaz G, Genova ML (2007) Kinetics of integrated electron transfer in the mitochondrial respiratory chain: random collisions vs. solid state electron channeling. Am J Physiol Cell Physiol 292:C12211239

Lenaz G, Genova ML (2012) Supramolecular organisation of the mitochondrial respiratory chain: a new challenge for the mechanism and control of oxidative phosphorylation. Adv Exp Med Biol 748:107-144

Lenaz G, Tioli G, Falasca Al, Genova ML (2016) Complex I function in mitochondrial supercomplexes. Biochim Biophys Acta 1857:991-1000

Leonard K, Haiker H, Weiss H (1987) Three-dimensional structure of NADH: ubiquinone reductase (complex I) from Neurospora mitochondria determined by electron microscopy of membrane crystals. J Mol Biol 194:277-286

Lepault J, Dubochet J, Baschong W, Kellenberger E (1987) Organization of double-stranded DNA in bacteriophages: a study by cryo-electron microscopy of vitrified samples. EMBO J 6:1507-1512

Letts JA, Fiedorczuk K, Degliesposti G, Skehel M, Sazanov LA (2019) Structures of respiratory supercomplex I+III2 reveal functional and conformational crosstalk. Mol Cell 75:1131-1146

Letts JA, Fiedorczuk K, Sazanov LA (2016) The architecture of respiratory supercomplexes. Nature 537:644-648

Letts JA, Sazanov LA (2017) Clarifying the supercomplex: the higher-order organization of the mitochondrial electron transport chain. Nat Struct Mol Biol 24:800-808 
Li X, Mooney P, Zheng S, Booth CR, Braunfeld MB, Gubbens S, Agard DA, Cheng $Y$ (2013) Electron counting and beam-induced motion correction enable near-atomic-resolution single-particle cryo-EM. Nat Methods 10:584-590

Liao M, Cao E, Julius D, Cheng Y (2013) Structure of the TRPV1 ion channel determined by electron cryo-microscopy. Nature 504:107-112

Lim Y-A, Rhein V, Baysang G, Meier F, Poljak AJ, Raftery M, Guilhaus M, Ittner LM, Eckert A, Götz J (2010) A $\beta$ and human amylin share a common toxicity pathway via mitochondrial dysfunction. Proteomics 10:1621-1633

Lobo-Jarne T, Ugalde C (2018) Respiratory chain supercomplexes: structures, function and biogenesis. Semin Cell Dev Biol 76:179190

Luo F, Gui X, Zhou H, Gu J, Li Y, Liu X, Zhao M, Li D, Li X, Liu C (2018) Atomic structures of FUS LC domain segments reveal bases for reversible amyloid fibril formation. Nat Struct Mol Biol 25:341-346

Maranzana E, Barbero G, Falasca Al, Lenaz G, Genova ML (2013) Mitochondrial respiratory supercomplex association limits production of reactive oxygen species from complex I. Antioxid Redox Signal 19:1469-1480

Marques I, Dencher NA, Videira A, Krause F (2007) Supramolecular organization of the respiratory chain in Neurospora crassa mitochondria. Eukaryot Cell 6:2391-2405

McMullan G, Faruqi AR, Clare D, Henderson R (2014) Comparison of optimal performance at $300 \mathrm{keV}$ of three direct electron detectors for use in low dose electron microscopy. Ultramicroscopy 147:156-163

McMullan G, Faruqi AR, Henderson R (2016) Direct electron detectors. Methods Enzymol 579:1-17

Melo AM, Bandeiras TM, Teixeira M (2004) New insights into type II $\mathrm{NAD}(\mathrm{P}) \mathrm{H}$ : quinone oxidoreductases. Microbiol Mol Biol Rev 68:603-616

Merk A, Bartesaghi A, Banerjee S, Falconieri V, Rao P, Davis MI, Pragani R, Boxer MB, Earl LA, Milne JLS et al (2016) Breaking cryo-EM resolution barriers to facilitate drug discovery. Cell 165:1698-1707

Milenkovic D, Blaza JN, Larsson N-G, Hirst J (2017) The enigma of the respiratory chain supercomplex. Cell Metab 25:765-776

Mileykovskaya E, Penczek PA, Fang J, Mallampalli VK, Sparagna GC, Dowhan W (2012) Arrangement of the respiratory chain complexes in Saccharomyces cerevisiae supercomplex III2IV2 revealed by single particle cryo-electron microscopy. J Biol Chem 287:23095-23103

Mitchell P (1961) Coupling of phosphorylation to electron and hydrogen transfer by a chemi-osmotic type of mechanism. Nature 191:144

Mitchell P (1975a) The protonmotive Q cycle: a general formulation. FEBS Lett 59:137-139

Mitchell P (1975b) Protonmotive redox mechanism of the cytochrome b-c1 complex in the respiratory chain: protonmotive ubiquinone cycle. FEBS Lett 56:1-6

Mitchell P (2011) Chemiosmotic coupling in oxidative and photosynthetic phosphorylation. Biochim Biophys Acta (BBA) 1807:1507-1538
Mourier A, Matic S, Ruzzenente B, Larsson NG, Milenkovic D (2014) The respiratory chain supercomplex organization is independent of COX7a2I isoforms. Cell Metab 20:1069-1075

Muller F, Crofts AR, Kramer DM (2002) Multiple Q-cycle bypass reactions at the Qo site of the cytochrome bc1 complex. Biochemistry 41:7866-7874

Nicholson WV, Glaeser RM (2001) Review: automatic particle detection in electron microscopy. J Struct Biol 133:90-101

Nogales E, Scheres SH (2015) Cryo-EM: a unique tool for the visualization of macromolecular complexity. Mol Cell 58:677-689

Nubel E, Wittig I, Kerscher S, Brandt U, Schagger H (2009) Twodimensional native electrophoretic analysis of respiratory supercomplexes from Yarrowia lipolytica. Proteomics 9:2408-2418

Ogura T, Sato C (2004) Automatic particle pickup method using a neural network has high accuracy by applying an initial weight derived from eigenimages: a new reference free method for single-particle analysis. J Struct Biol 145:63-75

Ohnishi T, Kawaguchi K, Hagihara B (1966) Preparation and some properties of yeast mitochondria. J Biol Chem 241:1797-1806

Ohnishi T, Ohnishi ST, Shinzawa-Itoh K, Yoshikawa S, Weber RT (2012) EPR detection of two protein-associated ubiquinone components $(\mathrm{SQ}(\mathrm{Nf})$ and $\mathrm{SQ}(\mathrm{Ns}))$ in the membrane in situ and in proteoliposomes of isolated bovine heart complex I. Biochim Biophys Acta 1817:1803-1809

Osuda Y, Shinzawa-Itoh K, Tani K, Maeda S, Yoshikawa S, Tsukihara T, Gerle C (2016) Two-dimensional crystallization of monomeric bovine cytochrome c oxidase with bound cytochrome c in reconstituted lipid membranes. Microscopy (Oxf) 65:263-267

Osyczka A, Moser CC, Daldal F, Dutton PL (2004) Reversible redox energy coupling in electron transfer chains. Nature 427:607-612

Osyczka A, Moser CC, Dutton PL (2005) Fixing the Q cycle. Trends Biochem Sci 30:176-182

Papa S, Capitanio G, Papa F (2018) The mechanism of coupling between oxido-reduction and proton translocation in respiratory chain enzymes. Biol Rev 93:322-349

Papa S, Martino PL, Capitanio G, Gaballo A, De Rasmo D, Signorile A, Petruzzella $\vee(2012)$ The oxidative phosphorylation system in mammalian mitochondria. In: Scatena R, Bottoni P, Giardina B (eds) Advances in mitochondrial medicine. Springer, Dordrecht, pp 3-37

Parey K, Brandt U, Xie H, Mills DJ, Siegmund K, Vonck J, Kuhlbrandt W, Zickermann V (2018) Cryo-EM structure of respiratory complex I at work. Elife 7:e39213

Penczek PA, Grassucci RA, Frank J (1994) The ribosome at improved resolution: new techniques for merging and orientation refinement in 3D cryo-electron microscopy of biological particles. Ultramicroscopy 53:251-270

Peng G, Fritzsch G, Zickermann V, Schagger H, Mentele R, Lottspeich F, Bostina M, Radermacher M, Huber R, Stetter KO et al (2003) Isolation, characterization and electron microscopic single particle analysis of the NADH:ubiquinone oxidoreductase (complex I) from the hyperthermophilic eubacterium Aquifex aeolicus. Biochemistry 42:3032-3039

Perez-Perez R, Lobo-Jarne T, Milenkovic D, Mourier A, Bratic A, Garcia-Bartolome A, Fernandez-Vizarra E, Cadenas S, Delmiro A, Garcia-Consuegra I et al (2016) COX7A2L is a mitochondrial complex III binding protein that stabilizes the III2+IV 
supercomplex without affecting respirasome formation. Cell Rep 16:2387-2398

Pieczenik SR, Neustadt J (2007) Mitochondrial dysfunction and molecular pathways of disease. Exp Mol Pathol 83:84-92

Pietras R, Sarewicz M, Osyczka A (2016) Distinct properties of semiquinone species detected at the ubiquinol oxidation Qo site of cytochrome bc1 and their mechanistic implications. J R Soc Interface. https://doi.org/10.1098/rsif.2016.0133

Pitceathly RDS, Taanman J-W (2018) NDUFA4 (Renamed COXFA4) is a cytochrome-c oxidase subunit. Trends Endocrinol Metab 29:452-454

Powell HR (2017) X-ray data processing. Biosci Rep. https://doi.org/ 10.1042/BSR20170227

Punjani A, Rubinstein JL, Fleet DJ, Brubaker MA (2017) cryoSPARC: algorithms for rapid unsupervised cryo-EM structure determination. Nat Methods 14:290-296

Radermacher M, Ruiz T, Clason T, Benjamin S, Brandt U, Zickermann V (2006) The three-dimensional structure of complex I from Yarrowia lipolytica: a highly dynamic enzyme. J Struct Biol 154:269-279

Radermacher M, Wagenknecht T, Verschoor A, Frank J (1987) Three-dimensional reconstruction from a single-exposure, random conical tilt series applied to the $50 \mathrm{~S}$ ribosomal subunit of Escherichia coli. J Microsc 146:113-136

Ragan Cl, Heron C (1978) The interaction between mitochondrial $\mathrm{NADH}$-ubiquinone oxidoreductase and ubiquinol-cytochrome $\mathrm{c}$ oxidoreductase. Evidence for stoicheiometric association. Biochem J 174:783-790

Ramirez-Aguilar SJ, Keuthe M, Rocha M, Fedyaev VV, Kramp K, Gupta KJ, Rasmusson AG, Schulze WX, van Dongen JT (2011) The composition of plant mitochondrial supercomplexes changes with oxygen availability. J Biol Chem 286:43045-43053

Rathore S, Berndtsson J, Marin-Buera L, Conrad J, Carroni M, Brzezinski P, Ott M (2019) Cryo-EM structure of the yeast respiratory supercomplex. Nat Struct Mol Biol 26:50-57

Razinkov I, Dandey V, Wei H, Zhang Z, Melnekoff D, Rice WJ, Wigge C, Potter CS, Carragher B (2016) A new method for vitrifying samples for cryoEM. J Struct Biol 195:190-198

Reifschneider NH, Goto S, Nakamoto H, Takahashi R, Sugawa M, Dencher NA, Krause F (2006) Defining the mitochondrial proteomes from five rat organs in a physiologically significant context using 2D blue-native/SDS-PAGE. J Proteome Res 5:1117-1132

Robinson AL (1986) Electron microscope inventors share nobel physics prize. Science 234:821-822

Rubinstein JL, Brubaker MA (2015) Alignment of cryo-EM movies of individual particles by optimization of image translations. J Struct Biol 192:188-195

Russo CJ, Passmore LA (2016) Progress towards an optimal specimen support for electron cryomicroscopy. Curr Opin Struct Biol 37:81-89

Sazanov LA (2015) A giant molecular proton pump: structure and mechanism of respiratory complex I. Nat Rev Mol Cell Biol 16:375-388

Sazanov LA, Baradaran R, Efremov RG, Berrisford JM, Minhas G (2013) A long road towards the structure of respiratory complex I, a giant molecular proton pump. Biochem Soc Trans 41:12651271

Schägger H, Pfeiffer K (2000) Supercomplexes in the respiratory chains of yeast and mammalian mitochondria. EMBO J 19:17771783

Schafer E, Dencher NA, Vonck J, Parcej DN (2007) Threedimensional structure of the respiratory chain supercomplex I1IIIIIV1 from bovine heart mitochondria. Biochemistry 46:12579-12585

Schafer E, Seelert H, Reifschneider NH, Krause F, Dencher NA, Vonck J (2006) Architecture of active mammalian respiratory chain supercomplexes. J Biol Chem 281:15370-15375

Schagger H, de Coo R, Bauer MF, Hofmann S, Godinot C, Brandt U (2004) Significance of respirasomes for the assembly/stability of human respiratory chain complex I. J Biol Chem 279:3634936353

Schagger H, Pfeiffer K (2001) The ratio of oxidative phosphorylation complexes I-V in bovine heart mitochondria and the composition of respiratory chain supercomplexes. J Biol Chem 276:3786137867

Schapira AHV (2006) Mitochondrial disease. The Lancet 368:70-82

Scharfe C, Lu HH-S, Neuenburg JK, Allen EA, Li G-C, Klopstock T, Cowan TM, Enns GM, Davis RW (2009) Mapping Gene Associations in human mitochondria using clinical disease phenotypes. PLoS Comput Biol 5:e1000374

Scheres SH (2012a) A Bayesian view on cryo-EM structure determination. J Mol Biol 415:406-418

Scheres SH (2012b) RELION: implementation of a Bayesian approach to cryo-EM structure determination. J Struct Biol 180:519-530

Scheres SH (2014) Beam-induced motion correction for submegadalton cryo-EM particles. Elife 3:e03665

Scheres SH (2016) Processing of structurally heterogeneous cryoEM data in RELION. Methods Enzymol 579:125-157

Scheres SH, Chen S (2012) Prevention of overfitting in cryo-EM structure determination. Nat Methods 9:853-854

Schur FK (2019) Toward high-resolution in situ structural biology with cryo-electron tomography and subtomogram averaging. Curr Opin Struct Biol 58:1-9

Sherer TB, Betarbet R, Greenamyre JT (2002) Environment, mitochondria, and Parkinson's disease. Neuroscientist 8:192197

Shi Y (2014) A glimpse of structural biology through X-ray crystallography. Cell 159:995-1014

Sigworth FJ (1998) A maximum-likelihood approach to singleparticle image refinement. J Struct Biol 122:328-339

Sousa JS, D'Imprima E, Vonck J (2018) Mitochondrial respiratory chain complexes. In: Harris JR, Boekema EJ (eds) Membrane protein complexes: structure and function. Springer, Singapore, pp 167-227

Sousa JS, Mills DJ, Vonck J, Kuhlbrandt W (2016) Functional asymmetry and electron flow in the bovine respirasome. Elife. https://doi.org/10.7554/eLife.21290

Standfuss J (2019) Membrane protein dynamics studied by X-ray lasers - or why only time will tell. Curr Opin Struct Biol 57:63-71 
Starkov AA, Fiskum G (2001) Myxothiazol induces $\mathrm{H}_{2} \mathrm{O}_{2}$ production from mitochondrial respiratory chain. Biochem Biophys Res Commun 281:645-650

Strauss M, Hofhaus G, Schroder RR, Kuhlbrandt W (2008) Dimer ribbons of ATP synthase shape the inner mitochondrial membrane. EMBO J 27:1154-1160

Strecker V, Wumaier Z, Wittig I, Schagger H (2010) Large pore gels to separate mega protein complexes larger than $10 \mathrm{MDa}$ by blue native electrophoresis: isolation of putative respiratory strings or patches. Proteomics 10:3379-3387

Stroh A, Anderka O, Pfeiffer K, Yagi T, Finel M, Ludwig B, Schagger H (2004) Assembly of respiratory complexes I, III, and IV into NADH oxidase supercomplex stabilizes complex I in Paracoccus denitrificans. J Biol Chem 279:5000-5007

Sun F, Huo X, Zhai Y, Wang A, Xu J, Su D, Bartlam M, Rao Z (2005) Crystal structure of mitochondrial respiratory membrane protein complex II. Cell 121:1043-1057

Taylor KA, Glaeser RM (1974) Electron diffraction of frozen, hydrated protein crystals. Science 186:1036-1037

Trouillard M, Meunier B, Rappaport F (2011) Questioning the functional relevance of mitochondrial supercomplexes by timeresolved analysis of the respiratory chain. Proc Natl Acad Sci USA 108:E1027-1034

Tsukihara T, Aoyama H, Yamashita E, Tomizaki T, Yamaguchi $\mathrm{H}$, Shinzawa-Itoh K, Nakashima R, Yaono R, Yoshikawa S (1995) Structures of metal sites of oxidized bovine heart cytochrome $\mathrm{C}$ oxidase at 2.8 A. Science 269:1069-1074

Tsukihara T, Aoyama H, Yamashita E, Tomizaki T, Yamaguchi $\mathrm{H}$, Shinzawa-ltoh K, Nakashima R, Yaono R, Yoshikawa S (1996) The whole structure of the 13-subunit oxidized cytochrome $\mathrm{C}$ oxidase at 2.8 A. Science 272:1136-1144

Turonova B, Schur FKM, Wan W, Briggs JAG (2017) Efficient 3DCTF correction for cryo-electron tomography using NovaCTF improves subtomogram averaging resolution to $3.4 \mathrm{~A}$. J Struct Biol 199:187-195

van Heel M, Frank J (1981) Use of multivariate statistics in analysing the images of biological macromolecules. Ultramicroscopy 6:187-194

van Heel M, Keegstra W (1981) IMAGIC: a fast, flexible and friendly image analysis software system. Ultramicroscopy 7:113-129

Vartak R, Porras CA-M, Bai Y (2013) Respiratory supercomplexes: structure, function and assembly. Protein Cell 4:582-590

Vempati UD, Han X, Moraes CT (2009) Lack of cytochrome c in mouse fibroblasts disrupts assembly/stability of respiratory complexes I and IV. J Biol Chem 284:4383-4391

Verner Z, Skodova I, Polakova S, Durisova-Benkovicova V, Horvath A, Lukes J (2013) Alternative NADH dehydrogenase (NDH2): intermembrane-space-facing counterpart of mitochondrial complex $\mathrm{I}$ in the procyclic Trypanosoma brucei. Parasitology 140:328-337

Vinothkumar KR, Zhu J, Hirst J (2014) Architecture of mammalian respiratory complex I. Nature 515:80

Vonck J (2012) Supramolecular organization of the respiratory chain

Wagner T, Merino F, Stabrin M, Moriya T, Antoni C, Apelbaum A, Hagel P, Sitsel O, Raisch T, Prumbaum D et al (2019) SPHIREcrYOLO is a fast and accurate fully automated particle picker for cryo-EM. Commun Biol 2:218
Wan W, Briggs JA (2016) Cryo-electron tomography and subtomogram averaging. Methods Enzymol 579:329-367

Wang F, Gong H, Liu G, Li M, Yan C, Xia T, Li X, Zeng J (2016) DeepPicker: a deep learning approach for fully automated particle picking in cryo-EM. J Struct Biol 195:325-336

Wang HW, Wang JW (2017) How cryo-electron microscopy and X-ray crystallography complement each other. Protein Sci 26:3239

Wang Y, Zhang SXL, Gozal D (2010) Reactive oxygen species and the brain in sleep apnea. Respir Physiol Neurobiol 174:307-316

Wharton DC, Tzagoloff A (1962) Studies on the electron transfer system. J Biol Chem 237:2051-2061

White HD, Thirumurugan K, Walker ML, Trinick J (2003) A second generation apparatus for time-resolved electron cryo-microscopy using stepper motors and electrospray. J Struct Biol 144:246-252

Wikstrom M, Sharma V, Kaila VR, Hosler JP, Hummer G (2015) New perspectives on proton pumping in cellular respiration. Chem Rev 115:2196-2221

Williams EG, Wu Y, Jha P, Dubuis S, Blattmann P, Argmann CA, Houten SM, Amariuta T, Wolski W, Zamboni N et al (2016) Systems proteomics of liver mitochondria function. Science 352: aad0189

Wiseman B, Nitharwal RG, Fedotovskaya O, Schafer J, Guo H, Kuang Q, Benlekbir S, Sjostrand D, Adelroth P, Rubinstein JL et al (2018) Structure of a functional obligate complex III2IV2 respiratory supercomplex from Mycobacterium smegmatis. Nat Struct Mol Biol 25:1128-1136

Wittig I, Braun HP, Schagger H (2006a) Blue native PAGE. Nat Protoc 1:418-428

Wittig I, Carrozzo R, Santorelli FM, Schagger H (2006b) Supercomplexes and subcomplexes of mitochondrial oxidative phosphorylation. Biochim Biophys Acta 1757:1066-1072

Wittig I, Schagger H (2009) Supramolecular organization of ATP synthase and respiratory chain in mitochondrial membranes. Biochim Biophys Acta 1787:672-680

Wong HC, Chen J, Mouche F, Rouiller I, Bern M (2004) Model-based particle picking for cryo-electron microscopy. J Struct Biol 145:157-167

Wright JJ, Salvadori E, Bridges HR, Hirst J, Roessler MM (2016) Small-volume potentiometric titrations: EPR investigations of Fe$\mathrm{S}$ cluster N2 in mitochondrial complex I. J Inorg Biochem 162:201-206

Wu M, Gu J, Guo R, Huang Y, Yang M (2016) Structure of mammalian respiratory supercomplex I1III2IV1. Cell 167:15981609.e1510

Xia D, Yu CA, Kim H, Xia JZ, Kachurin AM, Zhang L, Yu L, Deisenhofer J (1997) Crystal structure of the cytochrome bc1 complex from bovine heart mitochondria. Science 277:60-66

Yang XH, Trumpower BL (1986) Purification of a three-subunit ubiquinol-cytochrome c oxidoreductase complex from Paracoccus denitrificans. J Biol Chem 261:12282-12289

Yang YQ, Yu Y, Li XL, Li J, Wu Y, Yu J, Ge JP, Huang ZH, Jiang LB, Rao $Y$ et al (2017) Target elucidation by cocrystal structures of NADH-ubiquinone oxidoreductase of Plasmodium falciparum (PfNDH2) with small molecule to eliminate drug-resistant malaria. J Med Chem 60:1994-2005 
Yankovskaya V, Horsefield R, Tornroth S, Luna-Chavez C, Miyoshi H, Leger C, Byrne B, Cecchini G, Iwata S (2003) Architecture of succinate dehydrogenase and reactive oxygen species generation. Science 299:700-704

Yano T, Rahimian M, Aneja KK, Schechter NM, Rubin H, Scott CP (2014) Mycobacterium tuberculosis type II NADH-menaquinone oxidoreductase catalyzes electron transfer through a two-site ping-pong mechanism and has two quinone-binding sites. Biochemistry 53:1179-1190

Yoshikawa S, Shimada A (2015) Reaction mechanism of cytochrome c oxidase. Chem Rev 115:1936-1989

Yoshikawa S, Shinzawa-ltoh K, Nakashima R, Yaono R, Yamashita E, Inoue N, Yao M, Fei MJ, Libeu CP, Mizushima T et al (1998) Redox-coupled crystal structural changes in bovine heart cytochrome c oxidase. Science 280:1723

Zeviani M, Di Donato S (2004) Mitochondrial disorders. Brain 127:2153-2172

Zhang M, Mileykovskaya E, Dowhan W (2002) Gluing the respiratory chain together: cardiolipin is required for supercomplex formation in the inner mitochondrial membrane. J Biol Chem 277:4355343556

Zhang Z, Huang L, Shulmeister VM, Chi YI, Kim KK, Hung LW, Crofts AR, Berry EA, Kim SH (1998) Electron transfer by domain movement in cytochrome bc1. Nature 392:677-684

Zheng SQ, Palovcak E, Armache JP, Verba KA, Cheng Y, Agard DA (2017) MotionCor2: anisotropic correction of beam-induced motion for improved cryo-electron microscopy. Nat Methods 14:331-332

Zhu J, Vinothkumar KR, Hirst J (2016) Structure of mammalian respiratory complex I. Nature 536:354

Zivanov J, Nakane T, Scheres SHW (2019) A Bayesian approach to beam-induced motion correction in cryo-EM single-particle analysis. IUCrJ 6:5-17

Zong S, Gu J, Liu T, Guo R, Wu M, Yang M (2018a) UQCRFS1N assembles mitochondrial respiratory complex-III into an asymmetric 21-subunit dimer. Protein Cell 9:586-591

Zong S, Wu M, Gu J, Liu T, Guo R, Yang M (2018b) Structure of the intact 14-subunit human cytochrome c oxidase. Cell Res 28:1026-1034 